\title{
Spectral deformation of Laplacians on hyperbolic manifolds
}

\author{
ERIK BALSLEV
}

Let $(M, g)$ be a two-dimensional Riemannian manifold with constant curvature -1 outside a compact set. $M$ can be decomposed as $M=M_{0} \cup Z_{1} \cup$ $\cdots \cup Z_{m}$, where $M_{0}$ is compact and the cusps $Z_{j}$ are isometric to $S_{j} \times\left[a_{j}, \infty\right)$, where $S_{j}$ is the unit circle and the metric on $Z_{j}$ is given by $d s_{j}^{2}=y_{j}^{-2}\left(d x_{j}^{2}+\right.$ $\left.d y_{j}^{2}\right)$. This is the class of admissible surfaces with $m$ cusps considered by W. Müller $[\mathrm{M}]$. Let $\Delta$ be the Laplacian on $M$, a selfadjoint operator with domain equal to the Sobolev space $H^{2}(M)$. The spectrum of $\Delta$ consists of a continuous spectrum of multiplicity $m, \sigma_{c}(\Delta)=\left[\frac{1}{4}, \infty\right)$, a finite discrete spectrum contained in $\left[0, \frac{1}{4}\right.$ ) and a (possibly empty) finite or infinite set of eigenvalues $\left\{\kappa_{j}\right\}$ embedded in the continuous spectrum. It was proved by Selberg [Se] that for hyperbolic manifolds of constant negative curvature $\Gamma \backslash h$, where $h$ is the Poincaré halfplane and $\Gamma$ is a congruence subgroup of $P-S L_{2}(\mathbb{R})$, the Laplacian has infinitely many embedded eigenvalues $\kappa_{j}$, in fact they satisfy a Weyl law

$$
\sharp\left\{\kappa_{j} \leq R^{2}\right\}=\frac{R^{2} A}{4 \pi}+0(R \log R)
$$

where $A$ is the area of $\Gamma \backslash h$. Moreover, the spectral counting function $M(R)$ defined by

$$
M(R)=-\frac{1}{2 \pi} \int_{0}^{R} \frac{\phi^{\prime}\left(\frac{1}{2}+i t\right)}{\phi\left(\frac{1}{2}+i t\right)} d t
$$

where $\phi(s)=\operatorname{det} C(s)$ and $C(s)$ is the scattering matrix, satisfies $M(R)=$ $0(R \log R)$. Selberg then conjectured that for all discrete subgroups $\Gamma$ of $P-\mathrm{SL}_{2}(\mathbb{R})$ the spectral counting function $M(R)$ for $\Delta$ on $\Gamma \backslash h$ satisfies $M(R)=0\left(R^{2-\delta}\right)$ for some $\delta>0$.

In order to investigate this problem Phillips and Sarnak [P-S1]-[P-S3] introduced the method of perturbation of the Laplacian in the Teichmüller space of the manifold $\Gamma \backslash h$. Let the perturbed operators $\Delta(\varepsilon)$ have the expansion $\Delta(\varepsilon)=\Delta+\varepsilon L+0\left(\varepsilon^{2}\right)$ near $\varepsilon=0$ and let $\kappa(\varepsilon)=\kappa(0)+a_{1} \varepsilon+$ 
$a_{2} \varepsilon^{2}+0\left(\varepsilon^{3}\right)$ be the expansion of a simple embedded eigenvalue near $\varepsilon=0$. Then $a_{1}$ is real and $a_{2}$ is given by the formula.

$$
\operatorname{Im} a_{2}=\frac{-1}{2 r} \sum_{l=1}^{m}\left|\left(L u, E_{l}\left(\frac{1}{2}+i r\right)\right)\right|^{2}
$$

where $\kappa(0)=\frac{1}{4}+r^{2}$ and $E_{l}(s, z)$ is the generalized eigenfunction of $\Delta$ in the $l$ 'th cusp. If $\operatorname{Im} a_{2} \neq 0, \kappa(\varepsilon)$ is a resonance of $\Delta(\varepsilon)$ for small $\varepsilon \neq 0$. The method of proof of Phillips and Sarnak utilizes the Lax-Phillips scattering theory for automorphic wave equation [L-P], in particular the generator $B$ of the associated semigroup. The operator $\frac{1}{2} I+B$ has a purely discrete spectrum consisting of eigenvalues (embedded and discrete) as well as resonances of $\Delta$ and the point $\frac{1}{2}$. This permits the application of analytic perturbation theory to the family $\Delta(\varepsilon)$ yielding in particular the above formula for $\operatorname{Im} a_{2}$. This formula has been known in the physics literature of Schrödinger operators for a long time under the name of Fermi's Golden Rule. It was proved by $\mathrm{B}$. Simon [Si], utilizing the dilation-analytic theory of [B-C], which in that case made the problem accessible to analytic perturbation theory.

The identity of this formula in the Euclidean and hyperbolic cases suggests the possibility of proving it by the same method in both cases. The basic problem is the separation of the embedded eigenvalues from the continuous spectrum. In the Euclidean case the operator $-\Delta+V$ is transformed by a family of unitary operators induced by dilations in the independent variables. Analytic continuation in the scaling parameter leads to a rotation of the continuous spectrum away from the eigenvalues, successively turning resonances into discrete eigenvalues. The analogous $s$ in the hyperbolic case are dilations in the hyperbolic distance or, equivalently, power $U(\lambda)$ of the transformations independent variables. We use a simple version of Hunziker's modification of the dilation-analytic theory [Hu]. Since the continuous spectrum of $\Delta$ is entirely controlled by the 0 -th Fourier mode and since the exponentially decreasing cusp forms explode under complex power transformations, these operators should be restricted to the $y$-coordinates in each cusp in the 0 -th Fourier mode, as was pointed out to us by M. Zworski. The operators $\mathrm{s} U(\lambda)$ are defined in section 1, and it is proved that the continuous spectrum $\left[\frac{1}{4}, \infty\right)$ of $\Delta$ rotates around $\frac{1}{4}$ through the angle $-2 \operatorname{Arg} \lambda$ into the continuous spectrum $\sigma_{c}(\lambda)=\frac{1}{4}+\lambda^{-2} \overline{\mathbb{R}^{+}}$of the transformed Laplacian $\Delta(\lambda)=U(\lambda) \Delta U\left(\lambda^{-1}\right)$. The embedded eigenvalues are unchanged for $|\operatorname{Arg} \lambda|<\pi$, and so are the isolated eigenvalues for $|\operatorname{Arg} \lambda|<\frac{\pi}{2}$. Resonances become discrete eigenvalues after being crossed by $\sigma_{c}(\lambda)$ (Theorem 1.1). In terms of the parameter $s$, related to the spectral parameter $\mu$ by 
$\mu=s(1-s), \sigma_{c}(\lambda)$ in the $s$-plane is the line $\frac{1}{2}-i \operatorname{Arg} \lambda \cdot \mathbb{R}$, which rotates around $\frac{1}{2}$ through the angles $\operatorname{Arg} \lambda \in(-\pi, \pi)$. The embedded eigenvalues remain on the line $\frac{1}{2}+i \mathbb{R}$, the isolated eigenvalues remain in the interval $\left(\frac{1}{2}, 1\right]$ for $|\operatorname{Arg} \lambda|<\frac{\pi}{2}$ and the resonances turn into discrete eigenvalues of $\Delta(\lambda)$ located between $\frac{1}{2}+i \mathbb{R}$ and $\sigma_{c}(\lambda)$. The reason why $\sigma_{c}(\lambda)$ is the line $\frac{1}{2}-i \operatorname{Arg} \lambda \cdot \mathbb{R}$ becomes transparent when we observe that the Eisenstein series $E(s, z)$ transforms under $U_{\lambda}$ into $E(\lambda, s, z)$ with the leading terms for large $y_{j}$ in $Z_{j}$ equal to $\lambda^{\frac{1}{2}}\left(y_{j}^{\frac{1}{2}+i \sigma \lambda}+C(s) y_{j}^{\frac{1}{2}-i \sigma \lambda}\right.$ ), where $s=\frac{1}{2}+\lambda^{-1} i \sigma$ (cf. (2.18)). Precisely when $\operatorname{Arg} \sigma=-\operatorname{Arg} \lambda$ are both of these terms "almost" in $L^{2}(M)$, which is characteristic of points in the continuous spectrum. The transformed Eisenstein series $E(\lambda, s, z)$ are discussed in section 2, which also contains a deformation-analytic proof of the existence of analytic continuations of the scattering matrix $C(s)$ and the Eisenstein series $E(s, z)$ from Res $>1$ to $\mathbb{C} \backslash\left\{\frac{1}{2}\right\}$ which poles contained in the set of resolvent resonances, i.e. poles of the resolvents $(\Delta(\lambda)-s(1-s))^{-1}$ (Theorem 2.3).

In section 3 we establish the analytic continuation of the resolvent kernel $\mathcal{R}(s, z, w)$ from Res $>1$ to $\mathbb{C} \backslash\left\{\frac{1}{2}\right\}$ and the continuation $\tilde{R}(s)$ of the resolvent $R(s)$ up to Res $>\frac{1}{2}-\delta$, considered as an operator in $B\left(L_{\delta}^{2}(M), L_{-\delta}^{2}(M)\right)$. Thereby the results of section 2 are completed to obtain the identity of the poles of $C(s), E(s, z)$, and $\tilde{R}(s)$ with the resolvent resonances (Theorem 3.1).

The fact that the resonance $\rho=\frac{1}{2}+i \sigma$ becomes a discrete eigenvalue of $\Delta(\lambda)$ for $\operatorname{Arg} \lambda<-\operatorname{Arg} \sigma$ can be explained in terms of the asymptotics of the properties of resonance functions. The 0 -th Fourier coefficient of the resonance function with resonance $\rho$ is for large $y$ transformed into $\operatorname{Res} C(s)\left(y_{j}^{\frac{1}{2}-i \sigma \lambda}\right)_{j=1}^{m}$, which becomes a set of square-integrable eigenfunctions of $\Delta(\lambda)$ for $\operatorname{Arg} \lambda<-\operatorname{Arg} \sigma$ (Theorem 4.1), so the resonance $\rho$ becomes a discrete eigenvalue of $\Delta(\lambda)$, when $\sigma_{c}(\lambda)$ crosses $\rho$. Similarly, the properties of eigenfunctions corresponding to an isolated eigenvalue of $\Delta$ explain why $\mu$ ceases to be an eigenvalue of $\Delta(\lambda)$ for $|\operatorname{Arg} \lambda| \geqq \frac{\pi}{2}$, unless the eigenfunction is a cusp form in which case $\lambda$ remains an eigenvalue for $|\operatorname{Arg} \lambda|<\pi$. As a consequence of the separation of $\sigma_{c}(\lambda)$ from the embedded eigenvalues Fermi's Golden Rule is now proved (Theorem 5.3 and 5.4) by the proof of [Si] for the class of perturbations of $\Delta$ in Teichmüller space studied by Phillips and Sarnak [P-S1]. The transformation given there, which reduces the problem to the study of a family of metrics varying real analytically only in a compact set, is essential for our proof and perfectly suited for the application of the method of complex power transformations.

Phillips and Sarnak showed that the perturbation in Teichmüller space can be considered as a perturbation $g(\varepsilon)$ of the metric on the manifold 
$M$, such that $g(\varepsilon)$ deviates from $g(0)$ only in a compact set, independent of $\varepsilon$. This perturbation theory is valid for manifolds of constant negative curvature. The family $\Delta(\varepsilon)$ of Laplacians on $(M, g(\varepsilon))$ equal $\Delta$ outside a compact set for all $\varepsilon \in\left(-\varepsilon_{0}, \varepsilon_{0}\right)$. Therefore it is possible to transform $\Delta(\varepsilon)$ for all $\varepsilon$ by the same complex power, so that $\sigma_{c}(\Delta(\lambda, \varepsilon))=\frac{1}{4}+\lambda^{-2} \overline{\mathbb{R}}^{+}$for all $\varepsilon \in\left(-\varepsilon_{0}, \varepsilon_{0}\right)$, setting the stage for analytic perturbation theory.

The importance of the formula for $\operatorname{Im} a_{2}$ known as Fermi's Golden Rule is that if for a given eigenvalue $\kappa$ and some $l,\left(L u, E_{1}(\lambda) \neq 0\right.$, then the eigenvalue $\kappa$ gives rise to resonance. On the other hand, if $\kappa$ remains in the continuous spectrum under the perturbation in Teichmüller space, then as an important consequence this integral is 0 .

A different proof of Fermi's Golden Rule was given by Petridis [Pe2], utilizing the method of Faddeev [F] of analytically continuing the resolvent kernel together with the Lax-Phillips scattering theory [L-P]. Our method does not treat the point $\frac{1}{2}$. For a thorough analysis of this point see [Pe1].

\section{The analytic family of operators $\Delta(\lambda)$.}

Let $(M, g)$ be a two-dimensional Riemannian manifold with constant curvature -1 outside a compact set $M_{0} . M$ can be decomposed as

$$
M=M_{0} \cup Z_{1} \cup \cdots Z_{m}
$$

where $M_{0}$ is compact and

$$
Z_{j} \cong S_{j} \times\left[a_{j}, \infty\right), j=1, \cdots, m
$$

with $a_{j}>0$ and the metric on $Z_{j}$ is given by the line element

$$
d s_{j}^{2}=\frac{d x_{j}^{2}+d y_{j}^{2}}{y_{j}^{2}}
$$

where $\left(x_{j}, y_{j}\right) \in S_{j} \times\left[a_{j}, \infty\right)$ and $S_{j}$ is the unit circle.

We denote by $\Delta$ the unique self-adjoint Laplacian in $\mathrm{L}^{2}(M)$ with domain $H^{2}(M)$, the Sobolev space of order 2 on $M$, acting in $Z_{j}$ as $-y_{j}^{2}\left(\frac{\partial^{2}}{\partial x_{j}^{2}}+\frac{\partial^{2}}{\partial y_{j}^{2}}\right)$.

The spectrum $\sigma(\Delta)$ of $\Delta$ consists of

1. the essential spectrum $\sigma_{e}(\Delta)=\left[\frac{1}{4}, \infty\right)$. 
2. the embedded point spectrum $\sigma_{p}(\Delta)=\left\{\lambda_{1}<\lambda_{2}<\cdots<\lambda_{n}<\cdots\right\}$, a finite (possibly empty) or infinite set of eigenvalues of finite multiplicity with $\lambda_{1}>\frac{1}{4}$.

3. the discrete spectrum $\sigma_{d}(\Delta)=\left\{0=\mu_{1}<\mu_{1}<\cdots<\mu_{k}<\frac{1}{4}\right\}$, a finite set of eigenvalues of finite multiplicity.

4. possibly the eigenvalue $\frac{1}{4}$.

In order to study the spectrum in more detail we introduce a selfadjoint, analytic family of operators $\Delta(\lambda)$, defined through complex power transformations of $\Delta$ in the 0 -th Fourier mode.

Let $\varphi$ be a real-valued $C^{\infty}$-function on $(0, \infty)$ with $\varphi^{\prime} \geq 0$ and satisfying for fixed $K$ and $R, a<K<R$, with $a=\max \left\{1, a_{1} \cdots, a_{n}\right\}$

$$
\varphi(y)=\left\{\begin{array}{l}
0 \text { for } 0<y<K \\
1 \text { for } R<y<\infty
\end{array}\right.
$$

Let $\Psi_{\lambda}(y)=y^{1+(\lambda-1) \varphi(y)}, \lambda \in \mathbb{C}$. Then

$$
\Psi_{\lambda}^{\prime}(y)=\frac{d}{d y} \Psi_{\lambda}(y)=y^{(\lambda-1) \varphi(y)}\left[1+(\lambda-1)\left(\varphi(y)+\varphi^{\prime}(y) y \log y\right)\right]
$$

Let

$$
\alpha=\left[\max _{K \leq y \leq R}\left\{\varphi(y)+\varphi^{\prime}(y) y \log y\right\}\right]^{-1}
$$

Then for $1-\alpha<\lambda<\infty$ and for $\operatorname{Im} \lambda \neq 0$ we have $\Psi_{\lambda}^{\prime} \neq 0$.

For $\lambda \in(1-\alpha, \infty)$ and $a \leq c \leq K$ define the transform $U_{\lambda}^{0} f$ of $f \in$ $L^{2}\left(c, \infty ; y^{-2} d y\right)$ by

$$
\left(U_{\lambda}^{0} f\right)(y)=f_{\lambda}(y)=f\left(\Psi_{\lambda}(y)\right)\left(\Psi_{\lambda}^{\prime}(y)\right)^{\frac{1}{2}} y^{-(\lambda-1) \varphi(y)}
$$

Then

$$
\int_{c}^{\infty}\left|f_{\lambda}(y)\right|^{2} y^{-2} d y=\int_{c}^{\infty}\left|f\left(\Psi_{\lambda}(y)\right)\right|^{2} \Psi_{\lambda}^{2}(y) \Psi_{\lambda}^{\prime}(y) d y=\int_{c}^{\infty}|f(u)|^{2} u^{-2} d u
$$

so $\left\{U_{\lambda}^{0}\right\}_{\lambda>1-\alpha}$ is a family of unitary operators on $L^{2}\left(c, \infty ; y^{-2} d y\right)$.

The inverse $U_{\lambda}^{0-1}$ of $U_{\lambda}^{0}$ is given by

$$
\left(U_{\lambda}^{0-1} h\right)(y)=h\left(\alpha_{\lambda}(y)\right)\left(\Psi_{\lambda}^{\prime}\left(\alpha_{\lambda}(y)\right)\right)^{-\frac{1}{2}} \alpha_{\lambda}^{-1}(y) y
$$

where $\alpha_{\lambda}$ is the inverse of $\Psi_{\lambda}$ on $(0, \infty), \Psi_{\lambda}\left(\alpha_{\lambda}(y)\right)=y$. 
Consider the operator $L=-y^{2} \frac{d^{2}}{d y^{2}}$ in $L^{2}\left(c, \infty ; y^{-2} d y\right)$ with maximal domain $D(L)=\left\{f \in L^{2} \mid y^{2} \frac{d^{2}}{d y^{2}} f \in L^{2}\right\}$. We calculate $L(\lambda)=U_{\lambda}^{0} L U_{\lambda}^{0-1}$ and obtain

$$
\begin{aligned}
L(\lambda)= & -\left(\Psi_{\lambda}^{\prime}\right)^{-2} \Psi_{\lambda}^{2} \frac{d^{2}}{d y^{2}}+2\left\{\left(\Psi_{\lambda}^{\prime}\right)^{-3} \Psi_{\lambda}^{\prime \prime} \Psi_{\lambda}^{2}+\left(\Psi_{\lambda}^{\prime}\right)^{-2} \Psi_{\lambda}^{2} y^{-1}-\right. \\
& \left.\left(\Psi_{\lambda}^{\prime}\right)^{-1} \Psi_{\lambda}\right\} \frac{d}{d y}+\left\{\frac{5}{4}\left(\Psi_{\lambda}^{\prime}\right)^{-4}\left(\Psi_{\lambda}^{\prime \prime}\right)^{2} \Psi_{\lambda}^{2}+\frac{1}{2}\left(\Psi_{\lambda}^{\prime}\right)^{-3} \Psi_{\lambda}^{\prime \prime \prime} \Psi_{\lambda}^{2}\right. \\
& -2\left(\Psi_{\lambda}^{\prime}\right)^{-3} \Psi_{\lambda}^{\prime \prime} \Psi_{\lambda}^{2} y^{-1}+\left(\Psi_{\lambda}^{\prime}\right)^{-2} \Psi_{\lambda}^{\prime \prime} \Psi_{\lambda}-2\left(\Psi_{\lambda}^{\prime}\right)^{-2} \Psi_{\lambda}^{2} y^{-2} \\
& \left.+2\left(\Psi_{\lambda}^{\prime}\right)^{-1} \Psi_{\lambda} y^{-1}\right\}
\end{aligned}
$$

For $y>R, \Psi_{\lambda}(y)=y^{\lambda}$, and (1.3) reduces to

$$
(L(\lambda) h)(y)=\left(\left[\lambda^{-2}\left(L-\frac{1}{4}\right)+\frac{1}{4}\right] h\right)(y) \text { for } y>M
$$

For $y<K, \Psi_{\lambda}(y)=y$, so

$$
(L(\lambda) h)(y)=(L h)(y) \text { for } y<K
$$

We now define a family $U_{\lambda}$ of unitary operators on $L^{2}(M)$ as follows. On each cusp $Z_{j} \cong S^{1} \times\left[a_{j} ;, \infty\right), j=1 \cdots m$, let $f_{j} \in L^{2}\left(Z_{j}\right)$ have the Fourier expansion

$$
f_{j}(x, y)=\sum_{l \in \mathbf{Z}} a_{l}^{j}(y) e^{2 \pi i l x}
$$

and let

$$
\left(U_{\lambda}^{j} f\right)(x, y)=\left(U_{\lambda}^{0 j} a_{0}^{j}\right)(y)+\sum_{l}^{\prime} a_{l}^{j}(y) e^{2 \pi i l x}
$$

where $U_{\lambda}^{0 j}$ is defined by (1.1) and $\sum_{l}^{\prime}=\sum_{\mathbf{Z} \backslash\{0\}}$. Then $U_{\lambda}^{0 j}$ is unitary on $L^{2}\left(Z_{j}\right)$ for $j=1, \cdots, m, 1-\alpha<\lambda<\infty$.

For $f=\sum_{j=0}^{m} \oplus f_{j}, f_{0} \in L^{2}\left(M_{0}\right), f_{j} \in L^{2}\left(Z_{j}\right)$, let

$$
U_{\lambda} f=f_{0}+\sum_{j=1}^{m} U_{\lambda}^{j} f_{j}
$$


Here $\sum_{j=0}^{m} \oplus f_{j}$ is the function $f$ on $M$ such that $f\left|M_{0}=f_{0}, f\right| Z_{j}=f_{j}$ for $j=1 \cdots m$.

Then $\left\{U_{\lambda}\right\}_{\lambda>1-\alpha}$ is a family of unitary operators on $L^{2}(M)$. It follows from the definition of $U_{\lambda}$, that $U_{\lambda} \mathcal{D} \Delta \subset \mathcal{D}(\Delta)$. Then for $f \in \mathcal{D}(\Delta)$

$$
\Delta f=\sum_{j=0}^{m} \oplus \Delta_{j} f_{j}
$$

where $\Delta_{j}$ is the maximal operator corresponding to the formal Laplacian acting on $H^{2}\left(M_{0}\right)$ for $j=0$ and $H^{2}\left(Z_{j}\right)$ for $j=1 \cdots m$. The operators $\Delta_{j}$ act in $L^{2}\left(Z_{j} ; y_{j}^{-2} d y_{j}\right)$ by $\Delta_{j} f_{j}=\left(-y_{j}^{2}\left(\frac{\partial^{2}}{\partial x_{j}^{2}}+\frac{\partial^{2}}{\partial y_{j}}\right)\right) f_{j}$, where $\left(x_{j}, y_{j}\right)$ are coordinates on $Z_{j}$.

The operators $\Delta_{j}(\lambda)$ are defined for $\lambda \in(1-\alpha, \infty)$ by

$$
\begin{aligned}
& \Delta_{0}(\lambda)=\Delta_{0} \\
& \Delta_{j}(\lambda)=U_{\lambda}^{j} \Delta_{j}\left(U_{\lambda}^{j}\right)^{-1}
\end{aligned}
$$

By (1.7)

$$
\left(\Delta_{j}(\lambda)\right) f_{j}(x, y)=\left(L^{j}(\lambda) a_{0}^{j}(y)+\sum_{l}^{\prime}\left[\left(-y_{j}^{2} \frac{\partial^{2}}{\partial y_{j}^{2}}-(2 \pi l)^{2} y_{j}^{2}\right) a_{l}^{j}\right]\left(y_{j}\right) e^{2 \pi i l x_{j}}\right.
$$

where $L^{j}(\lambda)$ is given by (1.3) acting in $L^{2}\left(a_{j}, \infty ; y_{j}^{-2} d y_{j}\right)$.

The operators $\Delta(\lambda)$ with domain $\mathcal{D}(\Delta)$ are defined by

$$
\Delta(\lambda) f=\sum_{j=0}^{m} \oplus \Delta_{j}(\lambda) f_{j}
$$

The set $\{\Delta(\lambda)\}_{\lambda \in(1-\alpha, \infty)}$ is a family of selfadjoint operators with common domain $\mathcal{D}(\Delta)$, and each operator $\Delta(\lambda)$ is unitarily equivalent to $\Delta=\Delta(1)$ via the operator $U_{\lambda}$ defined by (1.1), (1.7) and (1.8).

The family $\{\Delta(\lambda)\}_{\lambda \in(1-\alpha, \infty)}$ has an analytic extension to $G_{\alpha}=\{\lambda \in \mathbb{C} \mid$ $\lambda \notin(-\infty, 1-\alpha]\}$, given by (1.3), (1.9) and (1.10), where $L(\lambda)$ given by (1.3), is defined and analytic for $\lambda \in G_{\alpha}$ with constant domain $\mathcal{D}(L(\lambda))=\mathcal{D}(L)$ since $\Psi_{\lambda}^{\prime}(y) \neq 0$ for $\lambda \in G_{\alpha}$ and $y>0$ and since $(L(\lambda) h)(y)$ is given for $y>R$ and $y<K$ by (1.4) and (1.5). The operators $\Delta(\lambda)$ satisfy $\Delta(\lambda)=\Delta^{\star}(\bar{\lambda})$ for $\lambda \in G_{\alpha}$, since $\left\{\Delta^{\star}(\bar{\lambda})\right\}_{\lambda \in G_{\alpha}}$ is analytic and $\Delta(\lambda)=\Delta^{\star}(\bar{\lambda})$ for $\lambda \in(1-\alpha, \infty)$. Thus, $\{\Delta(\lambda)\}_{\lambda \in G_{\alpha}}$ is a self-adjoint analytic family of type A, cf. [K]. 
For $\lambda$ non-real the essential spectrum $\sigma_{e}(\lambda)$ separates from the point spectrum $\sigma_{p}(\lambda)$. It follows from (1.4) applied to $L_{\lambda}^{j}, j=1 \cdots m$, that for $\lambda \in G_{\alpha}$

$$
\sigma_{c}(\lambda)=\frac{1}{4}+\lambda^{-2} \overline{\mathbb{R}^{+}}
$$

We refer to appendix 1 for the proof.

The set $\sigma_{p}(\lambda)$ of discrete eigenvalues in $\left(\frac{1}{4}, \infty\right)$ corresponds to cusp forms $u_{j}$, which remain constant under the action of $U_{\lambda}$, so $\sigma_{p}(\lambda)$ is constant for $\lambda \in G_{\alpha}$, equal to $\sigma_{p}(\Delta)$.

In appendix 2 we construct a dense set $\mathcal{F}$ of analytic vectors for $\left\{U_{\lambda}\right\}_{\lambda \in G_{\alpha}}$, i.e. for $f \in \mathcal{F}$ the function $U_{\lambda} f$ from $(1-\alpha, \infty)$ to $L^{2}(M)$ has an analytic extension to $\tilde{G}_{\alpha}=\left\{\lambda \in \mathbb{C}|| \operatorname{Arg} \lambda \mid<\frac{\pi}{2}, \lambda \notin(0,1-\alpha]\right\}$. As in [B-C] this implies that for every $f, g \in \mathcal{F}$ the function $\left(f,(\Delta-z)^{-1} g\right)$ has an analytic continuation from $\mathbb{C} \backslash \sigma(\Delta)$ across $\sigma_{c}(\Delta)$ with poles at resonances and eigenvalues. This in turn implies that the spectral function $(f, E(\lambda) f)$ is real analytic on $\mathbb{R} \backslash\left\{\sigma_{p}(\Delta) \cup \sigma_{d}(\Delta) \cup\left\{\frac{1}{4}\right\}\right\}$ for every $f \in \mathcal{F}$ and hence the absence of singular continuous spectrum of $\Delta$. Moreover, it follows that for $|\operatorname{Arg} \lambda|<\frac{\pi}{2}, \sigma_{d}(\lambda) \backslash \mathbb{R}$ is contained in the angle between the half-lines $\frac{1}{4}+\mathbb{R}^{+}$ and $\frac{1}{4}+\lambda^{-2} \mathbb{R}^{+}$and that $\sigma_{d}(\lambda) \cap \mathbb{R}=\sigma_{d}(\Delta)\left\{\frac{1}{4}\right\}$.

We summarize the basic spectral properties of $\Delta(\lambda)$ as follows.

Theorem 1.1. The set of operators $\{\Delta(\lambda)\}_{\lambda \in G_{\alpha}}$ is a self-adjoint family of type $A$ on $\mathcal{D}(\Delta(\lambda))=\mathcal{D}(\lambda)$.

The spectrum $\sigma(\lambda)$ of $\Delta(\lambda)$ satisfies for $\lambda \in G_{\alpha}$

1. The essential spectrum $\sigma_{e}(\lambda)$ is the half-line $\frac{1}{4}+\lambda^{-2} \overline{\mathbb{R}^{+}}$.

2. $\sigma_{d}(\lambda) \cap\left(\frac{1}{4}, \infty\right):=\sigma_{p}(\lambda)=\sigma_{p}(\Delta)$.

3. The singular continuous spectrum $\sigma_{s c}(\Delta)$ is empty.

4. For $|\operatorname{Arg} \lambda|<\frac{\pi}{2}, \sigma_{d}(\lambda) \backslash \mathbb{R}$ is contained in the angle between the halflines $\frac{1}{4}+\mathbb{R}^{+}$and $\frac{1}{4}+\lambda^{-2} \mathbb{R}^{+}$and is otherwise $\lambda$-independent (the set of resonances of $\Delta$ ).

5. $\sigma_{d}(\lambda) \cap\left(-\infty, \frac{1}{4}\right)=\sigma_{d}(\Delta)$ for $|\operatorname{Arg} \lambda|<\frac{\pi}{2}$. We shall return to 4 and 5 in section 3, where the restriction $|\operatorname{Arg} \lambda|<\frac{\pi}{2}$ will be removed.

\section{Transformation of the Eisenstein series $E(z, s)$..}

The set of generalized eigenfunctions given by the Eisenstein series $E(z, s)=\left\{E_{k}(z, s)\right\}_{k=1}^{m}$ has for Res $>1$ the following Fourier expansion 
in $Z_{j}$ for $y_{j}>a_{j}$,

$$
E_{k}(z, s)=\delta_{k}^{j} y_{j}^{s}+C_{k j}(s) y_{j}^{1-s}+\sum_{l}^{\prime} A_{k j}^{l}(s) y_{j}^{\frac{1}{2}} K_{s-\frac{1}{2}}\left(2 \pi|l| y_{j}\right) e^{2 \pi i l x_{j}}
$$

where $K_{s-\frac{1}{2}}(y)$ is the McDonald-Bessel function of order $s-\frac{1}{2}$ which decreases exponentially as $y \rightarrow \infty$ and $\sum_{l}^{\prime}$ is the sum over $l \neq 0$.

$E_{k}(z, s)$ is not in $L^{2}(M)$, but $E_{k}(z, s, \lambda):=U_{\lambda} E_{k}(z, s)$ is well defined and is given for Res $>1, \lambda \in(1-\alpha, \infty)$ and $z \in Z_{j}$ with $y_{j}>a_{j}$ by

$$
E_{k}(z, s, \lambda):=U_{\lambda} E_{k}(z, s)=\delta_{k}^{j} U_{\lambda}^{0 j} y_{j}^{s}+C_{k j}(s) U_{\lambda}^{0 j} y_{j}^{1-s}+\sum_{l}^{\prime}
$$

where $U_{\lambda}^{0 j} y_{j}^{s}$ is defined according to (1.1) for $\lambda \in(1-\alpha, \infty)$ and $y_{j}>a_{j}$ by

$$
U_{\lambda}^{0 j} y_{j}^{s}=y_{j}^{\left(1+(\lambda-1) \varphi\left(y_{j}\right)\right) s}\left(\Psi_{\lambda}^{\prime}\left(y_{j}\right)\right)^{1 / 2} y_{j}^{-(\lambda-1) \varphi(y)}
$$

It follows from (2.3) that $U_{\lambda}^{0 j} y_{j}^{s}$ and $U_{\lambda}^{0 j} y^{1-s}$ and hence $E_{k}(z, s, \lambda)$ for fixed $s$ with Res $>1$ and $z \in M$ have analytic extensions from $\lambda \in(1-\alpha, \infty)$ to $\lambda \in G_{\alpha}$, also given by (2.2) and (2.3).

For fixed $\lambda \in G_{\alpha}$ we obtain, following [CdV], the analytic continuation in $s$ of $E(z, s, \lambda)$ to $\left\{s \mid \operatorname{Re}\left\{\left(s-\frac{1}{2}\right) \lambda\right\}>0\right\}$.

Let $\alpha \in C^{\infty}\left(\mathbb{R}^{+}\right), 0 \leq \alpha \leq 1$, and assume

$$
\alpha(y)= \begin{cases}0 & \text { for } y<a \\ 1 & \text { for } y>a+1\end{cases}
$$

Define $\tilde{E}(z, s, \lambda)=\left\{\tilde{E}_{k}(z, s, k)\right\}_{k=1}^{m}$ on $Z_{k}$ for $y_{k}>a$ by

$$
\tilde{E}_{k}(z, s, \lambda)=E_{k}(z, s, \lambda)-U_{\lambda}^{0 k}\left(\alpha\left(y_{k}\right) y_{k}^{s}\right)
$$

where $K$ is chosen such that $K>a+1$, and on the rest of $M$ by $\tilde{E}_{k}(z, s, \lambda)=$ $E_{k}(z, s, \lambda)$.

For Res $>1$ and $\lambda \in(1-\alpha, \infty)$

$$
(\Delta(\lambda)-s(1-s)) E_{k}(z, s, \lambda)=0
$$

By analytic continuation this holds for Res $>1, \lambda \in G_{\alpha}$. This follows by integrating (2.5) with any function in $C_{0}^{\infty}(M)$ and using the analyticity properties of $\Delta(\lambda)$ and $E(z, s, \lambda)$. 
This implies for Res $>1, \lambda \in G_{\alpha}$

$$
(\Delta(\lambda)-s(1-s)) \tilde{E}_{k}(z, s, \lambda)=\Psi_{k}(z, s, \lambda),
$$

where

$$
\Psi_{k}(z, s, \lambda)=-(\Delta(\lambda)-s(1-s)) U_{\lambda}^{0 k}\left(\alpha\left(y_{k}\right) y_{k}^{s}\right) \text { for } z \in Z_{k}, y_{k}>a
$$

and $\Psi_{k}(z, s, \lambda)=0$ on the rest of $M$.

The functions $\Psi_{k}(z, s, \lambda)$ belong to $C_{0}^{\infty}(M)$ and are analytic in $s$ and $\lambda$ for $s \in \mathbb{C}, \lambda \in G_{\alpha}$. The resolvent $R(s, \lambda)=(\Delta(\lambda)-s(1-s))^{-1}$ is a meromorphic function of $s$ for $\lambda \in G_{\alpha}, \operatorname{Re}\left\{\left(s-\frac{1}{2}\right) \lambda\right\}>0$, by Theorem 1.1. Note that $\sigma_{e}(\lambda)$ in the $s$-plane is the line $\frac{1}{2}+\lambda^{-1} \mathrm{i} \mathbb{R}$. For $|\operatorname{Arg} \lambda|<\frac{\pi}{2}$ by Theorem 1.1 the poles of $R(s, \lambda)$ are contained in the angle between $\frac{1}{2}+i \mathbb{R}$ and $\frac{1}{2}+\lambda^{-1} i \mathbb{R}$ (the resonances), in $\frac{1}{2}+i \mathbb{R}$ (embedded eigenvalues of $\Delta$ ) and between $\frac{1}{2}$ and 1 (discrete eigenvalues of $\Delta$ ). Here we have identified $s$ with $s(1-s)$ in the notation $R(s, \lambda)=R(s(1-s), \lambda)$. This yields an $L^{2}(M)$-valued analytic continuation of $\tilde{E}_{k}(\bullet, s, \lambda)$ defined for $\lambda \in G_{\alpha}, \operatorname{Re}\left\{\left(s-\frac{1}{2}\right) \lambda\right\}>0$ by

$$
\tilde{E}_{k}(\bullet, s, \lambda)=R(s, \lambda) \Psi_{k}(\bullet, s, \lambda)
$$

This function is meromorphic in $s$ for each fixed $\lambda$ with poles contained in the set of poles of $R(s, \lambda)$.

Since $\tilde{E}_{k}(\bullet, s, \lambda)$ is analytic with values in $H^{2}(M)$ and $H^{2}(M)$ is continuously embedded in $C(K)$ for every compact $K \subset M, \tilde{E}_{k}(\bullet, s, \lambda)$ can be developed in a power series convergent in $C(K)$ around each $\left(s_{0}, \lambda_{0}\right)$ such that $s_{0}$ is not a pole of $\tilde{E}_{k}\left(\bullet, s, \lambda_{0}\right)$. It follows that for fixed $z \in M$ and $\lambda \in G_{\alpha}, \tilde{E}_{k}(z, s, \lambda)$ is meromorphic in $s$ for $\operatorname{Re}\left\{\left(s-\frac{1}{2}\right) \lambda\right\}>0$ with poles contained in the set of poles of $R(s, \lambda)$. Since $U_{\lambda}^{0 j}\left(\alpha\left(y_{j}\right) y_{j}^{s}\right)$ is analytic in $\lambda$ and $s$, we obtain an analytic continuation of $E_{k}(z, s, \lambda)$ given by $(2.4)$ and we have proved the following result.

Theorem 2.1. For fixed $z \in M$ and $\lambda \in G_{\alpha}, E(z, s, \lambda)$ is meromorphic in $s$ for $\operatorname{Re}\left\{\left(s-\frac{1}{2}\right) \lambda\right\}>0$ with poles contained in the set of poles of $R(s, \lambda)$. If $s$ is not a resonance or eigenvalue of $\Delta, E(z, s, \lambda)$ is analytic in $\lambda$ for $|\operatorname{Arg} \lambda|<\frac{\pi}{2}$.

We shall now use the above result to establish the analytic continuation of $C(s)$ and $A^{l}(s)$ from Res> 1 to $\mathbb{C} \backslash\left\{\frac{1}{2}\right\}$. It follows from the above definition of $\tilde{E}_{k}(z, s, \lambda)$, that these functions and hence the generalized eigenfunctions 
$E_{k}(z, s, \lambda)$ are 1-periodic in $x_{j}$ for $y_{j}>a_{j}$ in each cusp $Z_{j}$. Thus for $\operatorname{Re}\{(s-$ $\left.\left.\frac{1}{2}\right) \lambda\right\}>0, E_{k}(z, s, \lambda)$ has a Fourier expansion in $Z_{j}$ of the form

$$
\begin{aligned}
E_{k}(z, s, \lambda)= & \delta_{j}^{k} U_{\lambda}^{0 j}\left(y_{j}^{s}\right)+C_{k j}(s, \lambda) U_{\lambda}^{0 j}\left(y_{j}^{1-s}\right) \\
& +\sum_{l}^{\prime} A_{k j}^{l}(s, \lambda) y_{j}^{\frac{1}{2}} K_{s-\frac{1}{2}}\left(2 \pi|l| y_{j}\right) e^{2 \pi i l x_{j}}
\end{aligned}
$$

where $K_{s-\frac{1}{2}}\left(y_{j}\right)$ must decrease exponentially, since by construction

$$
E_{k}(\bullet, s, \lambda)-\delta_{j}^{k} U_{\lambda}^{0 j}\left(y_{j}^{s}\right) \in L^{2}(M) .
$$

Since $E_{k}(z, s, \lambda)$ is analytic in $s$ and $\lambda$ for each $z \in M$ by Theorem 2.1, each Fourier coefficient is analytic in $s$ and $\lambda$. Since $U_{\lambda}^{0 j}\left(y_{j}^{s}\right)$ are analytic in $s$ and $\lambda$ and $K_{s-\frac{1}{2}}\left(2 \pi|l| y_{j}\right)$ is analytic in $s$, it follows that $C_{k j}(s, \lambda)$ and $A_{k j}^{l}(s, \lambda)$ are analytic in $s$ and $\lambda$. For Res $>1, E_{k}(z, s, \lambda)$ are given by (2.2) as well as (2.7), hence $C_{k j}(s, \lambda)=C_{k j}(s)$ and $A_{k j}^{l}(s, \lambda)=A_{k j}^{l}(s)$ are independent of $\lambda \in G_{\alpha}$. By analytic continuation this holds for all $s$ with $\operatorname{Re}\left\{\left(s-\frac{1}{2}\right) \lambda\right\}>0$. We have proved

Lemma 2.2. $C(s)$ and $A^{l}(s)$ have analytic continuations from Res $>1$ to $\left\{s \| \operatorname{Arg}\left(s-\frac{1}{2}\right) \mid<\frac{3 \pi}{2}\right\}$ with poles contained in the union of the sets $\sigma_{d}(\Delta) \subset$ $\left(\frac{1}{2}+1\right], \sigma_{p}(\Delta) \subset \frac{1}{2}+i \mathbb{R}$ and $\bigcup_{\lambda \in G_{\alpha}} r(\lambda)$, where $r(\lambda)$ is the set of resonances (discrete eigenvalues of $\Delta(\lambda)$ ) contained in the angle between $\frac{1}{2}+i \mathbb{R}$ and $\frac{1}{2}+\lambda^{-1} i \mathbb{R}$.

We shall now show, that the analytic continuations of $C(s)$ and $A^{l}(s)$ from Res $>\frac{1}{2}$ around $\frac{1}{2}$ in the two directions agree for $s \in\left(-\infty, \frac{1}{2}\right)$.

Consider the Eisenstein series $E(z, s)$. By Lemma 2.2, $E(z, s)$ has an analytic continuation in $s$ given for $z \in Z_{j}$ by (2.1) or, in matrix form

$$
\begin{aligned}
E(z, s)= & \left(y_{k}^{s}\right)_{k=1}^{m}+C(s)\left(y_{j}^{1-s}\right)_{j=1}^{m} \\
& +\sum_{l}^{\prime} A^{l}(s)\left(y_{j}^{\frac{1}{2}} K_{s-\frac{1}{2}}\left(2 \pi|l| y_{j}\right) e^{2 \pi i l x_{j}}\right)_{j=1}^{m}
\end{aligned}
$$

Applying $C(1-s)$ to $E(z, s)$, given by (2.8), we get on replacing $s$ by $1-s$

$$
C(s) E(z, 1-s)=C(s) C(1-s)\left(y_{j}^{s}\right)_{j=1}^{m}+C(s)\left(y_{j}^{1-s}\right)_{j=1}^{m}+\sum_{l}^{\prime}
$$


Subtracting (2.9) from (2.8), we get

$$
E(z, s)-C(s) E(z, 1-s)=(I-C(s) C(1-s))\left(y_{j}^{s}\right)_{j=1}^{m}+\sum_{l}^{\prime}
$$

The l.h.s. of (2.11) satisfies

$$
(\Delta-s(1-s))[E(z, s)-C(s) E(z, 1-s)]=0
$$

For Res $<\frac{1}{2}$, the r.h.s of $(2.10)$ is in $L^{2}(M)$. Since $s(1-s)$ is not an eigenvalue of $\Delta$, it follows that the function given by the r.h.s. side of (2.10) is equal to 0 and hence if $s$ is not a pole of $C(s)$ or $C(1-s)$

$$
C(s) C(1-s)=I \text { for }\left|\operatorname{Arg}\left(s-\frac{1}{2}\right)\right|<\frac{3 \pi}{2} \text {. }
$$

In particular, if $\frac{1}{2}+\rho$ is not an eigenvalue of $\Delta$,

$$
C\left(\frac{1}{2}+\rho e^{i \pi}\right)=C\left(\frac{1}{2}+\rho e^{-i \pi}\right)=\left(C\left(\frac{1}{2}+\rho\right)\right)^{-1} \text { for } \rho>0
$$

so the two analytic continuations of $C(s)$ around $\frac{1}{2}$ agree.

Moreover, the l.h.s. of (2.11) equal to 0 yields for $s$ not a pole of $C(s)$ or $C(1-s)$

$$
E(z, s)=C(s) E(z, 1-s), s \in \mathbb{C} \backslash\{0\}
$$

For $\lambda>1$ and $s>1$, the function $\Psi_{k}(z, s, 1)$ defined by $(2.6)$ is real-valued, and $R(s, 1)=(\Delta-s(\Delta-s(1-s)))^{-1}$ is self-adjoint and real (maps real functions into real functions), so $\tilde{E}_{k}(z, s, 1)$ and hence $E_{k}(z, s)=E_{k}(z, s, 1)$ is real-valued. This implies that $C(s)$ is real for $s>1$ and hence $C(\bar{s})=\overline{C(s)}$ for $s \in \mathbb{C} \backslash\left\{\frac{1}{2}\right\}$.

Let

$$
\tilde{\tilde{E}}_{k, a}(z, s)= \begin{cases}E_{k}(z, s)-\left[\delta_{k}^{j}\left(y_{j}\right)^{s}+C_{k j} y_{j}^{1-s}\right] & \text { for } y_{j}>a, j=1 \cdots m \\ E_{k}(z, s) & \text { on the rest of } M\end{cases}
$$

By the Maass-Selberg relation [Se], for $s_{1} \neq \bar{s}_{2}, s_{1}+\bar{s}_{2} \neq 1$.

$$
\begin{aligned}
\int_{M} \tilde{\tilde{E}}_{k, a}\left(z, s_{1}\right) \tilde{\tilde{E}}_{j, a}\left(z, s_{2}\right) d g(z)= & \frac{\delta_{k}^{j} a^{s_{1}+\bar{s}_{2}-1}-a^{1-\left(s_{1}+\bar{s}_{2}\right)} \sum_{i=1}^{m} C_{k i}\left(s_{1}\right) \bar{C}_{j i}(s)}{s_{1}+\bar{s}_{2}-1} \\
& +\frac{\bar{C}_{j k}\left(s_{2}\right) a^{s_{1}-\bar{s}_{2}}-C_{k j}\left(s_{1}\right) a^{\bar{s}_{2}-s_{1}}}{s_{1}-\bar{s}_{2}} .
\end{aligned}
$$


For $s_{1}>s_{2}>1$ the l.h.s and the first term on the r.h.s. have finite limits as $s_{2} \rightarrow s_{1}$, hence the last term has a limit, and it follows that $\bar{C}_{k j}\left(s_{1}\right)=C_{j k}\left(s_{1}\right)$. Since $\bar{C}_{k j}\left(s_{1}\right)=C_{k j}\left(s_{1}\right)$, we obtain $C_{k j}\left(s_{1}\right)=C_{j k}\left(s_{1}\right)$ for $s_{1}>1$. By analytic continuation

$$
C_{j k}(s)=C_{k j}(s)=\bar{C}_{j k}(\bar{s}) \text { for } s \in \mathbb{C} \backslash\{0\} .
$$

In matrix notation

$$
C(s)=C^{t}(s)=C^{*}(\bar{s})
$$

In particular, for $s=\frac{1}{2}=i \sigma, \sigma \in \mathbb{R}-\{0\}$

$$
C\left(\frac{1}{2}+i \sigma\right)=C^{-1}\left(\frac{1}{2}-i \sigma\right)=C^{*}\left(\frac{1}{2}-i \sigma\right)
$$

so $C(s)$ is unitary on the line $\frac{1}{2}+i \mathbb{R}$ except $\frac{1}{2}$. It follows that $C(s)$ has no poles on this line including $\sigma_{p}(\Delta)$ and $\frac{1}{2}$.

To exclude an essential singularity at $\frac{1}{2}$ requires an estimate of the resolvent near $\frac{1}{2}$ to show that $E(z, s)$ and hence $C(s)$ is bounded near $\frac{1}{2}$. It is desirable and should be possible to obtain a simple proof of this well known fact in the present context.

We have proved the following result.

Theorem 2.3. The scattering matrix $C(s)$, the coefficients $A^{l}(s)$ and the Eisenstein series $E(z, s), z$ fixed in $M$, have analytic continuations to $\mathbb{C} \backslash\left\{\frac{1}{2}\right\}$ with poles contained in the union of the sets $\sigma_{d}(\Delta) \subset\left(\frac{1}{2}, 1\right], \sigma_{p}(\Delta) \subset \frac{1}{2}+i \mathbb{R}$ and $\bigcap_{\varepsilon>0}\left\{\bigcup\left\{r(\lambda)|| \operatorname{Arg} \lambda \mid<\frac{\pi}{2}+\varepsilon\right\}\right.$. They satisfy the following equations for $z \in M, s \in \mathbb{C} \backslash\{0\}, s$ not a pole of $C(s)$ or $C(1-s)$.

$$
\begin{gathered}
C(s) C(1-s)=1 \\
C(s)=\overline{C(\bar{s})}=C^{t}(s)=C^{*}(\bar{s}) \\
E(z, s)=C(s) E(z, 1-s)
\end{gathered}
$$

Consider now the Eisenstein series $E_{k}(z, s, \lambda)$ defined in $Z_{j}$ by (2.2) for $s \in \mathbb{C} \backslash\left\{\frac{1}{2}\right\}$ and $\lambda \in G_{\alpha}$, where $C(s)$ and $A^{l}(s)$ are the meromorphic functions in $\mathbb{C} \backslash\left\{\frac{1}{2}\right\}$ given by Theorem 2.3.

From (2.13) follows by applying $U_{\lambda}$ for $\lambda \in(1-\alpha, \infty)$

$$
E(z, s, \lambda)=C(s) E(z, 1-s, \lambda)
$$

and hence by analytic continuation (2.14) holds for $\lambda \in G_{\alpha}, s \in C \backslash\left\{\frac{1}{2}\right\}$. 
The $\lambda$-dependence of $E(z, x, \lambda)$ is in the first two terms. $U_{\lambda}^{0 j} y_{j}^{s}$ is given according to (1.1) by

$$
U_{\lambda}^{0 j} y_{j}^{s}=y_{j}^{\left(1+(\lambda-1) \varphi\left(y_{j}\right)\right) s}\left(\Psi_{\lambda}^{\prime}\left(y_{j}\right)\right)^{1 / 2} y_{j}^{-(\lambda-1) \varphi\left(y_{j}\right)}
$$

By definition of $\Psi_{\lambda}$, for $y_{j} \leq K, U_{\lambda}^{0 j} y_{j}^{s}=y_{j}^{s}$ and for $y_{j}>R$

$$
\begin{gathered}
U_{\lambda}^{0 j} y_{j}^{s}=\lambda^{1 / 2} y_{j}^{\frac{1}{2}+\lambda\left(s-\frac{1}{2}\right)} \\
U_{\lambda}^{0 j} y_{j}^{1-s}=\lambda^{1 / 2} y_{j}^{\frac{1}{2}-\lambda\left(s-\frac{1}{2}\right)}
\end{gathered}
$$

The essential spectrum $\sigma_{c}(\lambda)$ of $\Delta(\lambda)$ is the line $\frac{1}{2}+\lambda^{-1} i \mathbb{R}=\{s \mid \lambda(s-$ $\left.\left.\frac{1}{2}\right) \in i \mathbb{R}\right\} ;$ for $s=\frac{1}{2}+\lambda^{-1} i \sigma, \sigma \in \mathbb{R}$, and $y_{j}>R$

$$
U_{\lambda}^{0 j} y_{j}^{s}=\lambda^{1 / 2} y_{j}^{\frac{1}{2}+i \sigma}, U_{\lambda}^{0 j} y_{j}^{1-s}=\lambda^{1 / 2} y_{j}^{\frac{1}{2}-i \sigma}
$$

so $\left|E_{k}(z, s, \lambda)\right| \sim c y_{k}^{\frac{1}{2}}$ for $y_{k} \rightarrow \infty$ in $Z_{k}$.

Thus, as for $\lambda=1$, the continuous spectrum of $\Delta(\lambda)$ is the set of points $s$ such that $E(z, s, \lambda)$ is "almost" in $L^{2}(M)$.

The poles of $C(s)$ are contained in the union of the sets of resonances $\bigcup_{\lambda} r(\lambda)$ and $\sigma_{d}(\Delta)$. The set of poles of $C(s)$ in $S_{\frac{1}{2}}=\left\{s \mid 0<\right.$ Res $\left.<\frac{1}{2}\right\}$ in fact coincides with $\left(\bigcup_{\lambda}(r(\lambda)) \cap S_{\frac{1}{2}}\right)$. This will be proved in section 3 .

\section{Analytic continuation of the resolvent.}

It is well-known (cf. [M]) that each of the two sets of generalized eigenfunctions $\left\{\left\{E_{k}\left(\frac{1}{2} \pm i t\right)\right\}_{k=1}^{m} \mid t \in \mathbb{R}^{+}\right\}$yields a spectral resolution of the continuous part of $\Delta$.

For $f \in \mathcal{H}_{c}(\Delta) \cap C_{0}^{\infty}(M), g \in C_{0}^{\infty}(M)$

$$
(f, g)=\frac{1}{2 \pi} \sum_{j=1}^{m} \int_{0}^{\infty}\left(f, E_{j}\left(\bullet, \frac{1}{2}+i t\right)\right)\left(E_{j}\left(\bullet, \frac{1}{2}+i t\right), g\right) d t
$$

and

$$
(\Delta f, g)=\frac{1}{2 \pi} \sum_{j=1}^{m} \int_{0}^{\infty}\left(\frac{1}{4}+t^{2}\right)\left(f, E_{j}\left(\bullet, \frac{1}{2}+i t\right)\right)\left(E_{j}\left(\bullet, \frac{1}{2}+i t\right), g\right) d t
$$


Here $\mathcal{H}_{c}(\Delta)$ is the continuous subspace of $\mathrm{L}^{2}(M ; g)$ with respect to $\Delta$. by

For Res $>\frac{1}{2}, s \notin \sigma_{d}(\Delta)$, the resolvent $R(s)=(\Delta-s(1-s))^{-1}$ is given (3.3)

$$
\begin{aligned}
(R(s) f, g)= & \frac{1}{2 \pi} \sum_{j=1}^{m} \int_{0}^{\infty}\left(\frac{1}{4}+t^{2}-s(1-s)\right)^{-1} \\
& \left(f, E_{j}\left(\bullet, \frac{1}{2}+i t\right)\right)\left(E_{j}\left(\bullet, \frac{1}{2}+i t\right), g\right) d t
\end{aligned}
$$

Changing variables to $\lambda=s(1-s)$, the integral becomes

$$
\begin{array}{r}
I_{j}\left(\mu_{0} \pm i \varepsilon\right)=\int_{\frac{1}{4}}^{\infty}\left(\mu-\mu_{0} \mp i \varepsilon\right)^{-1}\left(f, E_{j}\left(\bullet, \frac{1}{2}+i t\right)\right) \\
\left(E_{j}\left(\bullet, \frac{1}{2}+i t\right), g\right) \frac{1}{2}\left(\mu-\frac{1}{4}\right)^{-\frac{1}{2}} d \mu
\end{array}
$$

where $t=\left(\mu-\frac{1}{4}\right)^{\frac{1}{2}}, s(1-s)=\mu_{0} \pm i \varepsilon$.

From (3.4) we obtain (cf. Appendix 3.(1))

$$
\begin{aligned}
I_{j}\left(\mu_{0} \pm i 0\right):= & \lim _{\varepsilon \downarrow 0} I_{j}\left(\mu_{0} \pm i \varepsilon\right) \\
= & v \cdot p \int_{\frac{1}{4}}^{\infty}\left(\mu-\mu_{0}\right)^{-1}\left(f, E_{j}\left(\bullet, \frac{1}{2}+i t\right)\right) \\
& \left(E_{j}\left(\bullet, \frac{1}{2}+i t\right), g\right) \frac{1}{2}\left(\mu-\frac{1}{4}\right)^{-\frac{1}{2}} d \mu \\
& \pm \pi i\left(f, E_{j}\left(\bullet, \frac{1}{2}+i\left(\mu_{0}-\frac{1}{4}\right)^{\frac{1}{2}}\right)\right) \\
& \left(E_{j}\left(\bullet, \frac{1}{2}+i\left(\mu_{0}-\frac{1}{4}\right)^{\frac{1}{2}}\right), g\right) \frac{1}{2}\left(\mu_{0}-\frac{1}{4}\right)^{-\frac{1}{2}}
\end{aligned}
$$

By (3.3) and (3.5)

$$
\left(f, R\left(\mu_{0} \pm i 0\right) g\right):=\lim _{\varepsilon \downarrow 0}\left(f, R\left(\mu_{0} \pm i \varepsilon\right) g\right)=\frac{1}{2 \pi} \sum_{j=1}^{m} I\left(\mu_{0} \pm i 0\right)
$$


It follows from (2.1) that $E_{j}\left(\bullet, \frac{1}{2}+i t\right) \in L_{-\delta}^{2}(M)$ for every $\delta>0$. This implies that $I\left(\mu_{0} \pm i \varepsilon\right)$ is defined and (3.5) holds for $f, g \in L_{\delta}^{2}(M), f \in \mathcal{H}_{c}(\Delta)$.

Hence $R\left(\mu_{0} \pm i o\right)$ is defined by (3.6) as an operator in $\mathcal{B}\left(L_{\delta}^{2}(M), \mathrm{L}_{-\delta}^{2}(M)\right)$ and $R\left(\mu_{0} \pm i 0\right)=w-\lim _{\varepsilon \downarrow 0} R\left(\mu_{0} \pm i \varepsilon\right)$. The kernel $\mathcal{R}\left(\mu_{0} \pm i 0 ; z, w\right)$ of $R(\mu \pm i 0)$ is given by

$$
\begin{aligned}
\mathcal{R}\left(\mu_{0} \pm i 0 ; z, w\right):= & \frac{1}{2 \pi} \sum_{j=1}^{m} v \cdot p \int_{\frac{1}{4}}^{\infty}\left(\mu-\mu_{0}\right)^{-1} \frac{1}{2}\left(\mu-\frac{1}{4}\right)^{-\frac{1}{2}} \\
& E_{j}\left(z, \frac{1}{2}+i\left(\mu-\frac{1}{4}\right)^{\frac{1}{2}}\right) \bar{E}_{j}\left(w, \frac{1}{2}+i\left(\mu-\frac{1}{4}\right)^{\frac{1}{2}}\right) d \mu \\
\pm & i \frac{1}{2}\left(\mu_{0}-\frac{1}{4}\right)^{-\frac{1}{2}} E_{j}\left(z, \frac{1}{2}+i\left(\mu-\frac{1}{4}\right)^{\frac{1}{2}}\right) \\
& \bar{E}_{j}\left(w, \frac{1}{2}+i\left(\mu-\frac{1}{4}\right)^{\frac{1}{2}}\right)
\end{aligned}
$$

From (3.7) we obtain the kernel $\mathcal{E}\left(\mu_{0} ; z, w\right)$ of the spectral density $P^{\prime}\left(\mu_{0}\right)$, given by

$$
\begin{aligned}
& \mathcal{E}\left(\mu_{0} ; z, w\right)= \frac{1}{2 \pi i}\left(\mathcal{R}\left(\mu_{0}+i 0 ; z, w\right)-\mathcal{R}\left(\mu_{0}-i 0 ; z, w\right)\right) \\
&= \frac{1}{2 \pi}\left(\mu_{0}-\frac{1}{4}\right)^{-\frac{1}{2}} \sum_{j=1}^{m} E_{j}\left(z, \frac{1}{2}+i\left(\mu_{0}-\frac{1}{4}\right)^{\frac{1}{2}}\right) \\
& \bar{E}_{j}\left(w, \frac{1}{2}+i\left(\mu_{0}-\frac{1}{4}\right)^{\frac{1}{2}}\right)
\end{aligned}
$$

In terms of the variable $t=\left(\mu-\frac{1}{4}\right)^{\frac{1}{2}}$, we have for $t \in \mathbb{R} \backslash\{0\}$

$$
\mathcal{R}\left(\frac{1}{2}+i t ; z, w\right)=\mathcal{R}\left(\frac{1}{2}-i t ; z, w\right)+i t^{-1} E_{j}\left(z, \frac{1}{2}+i t\right) \bar{E}_{j}\left(w, \frac{1}{2}+i t\right)
$$

The resolvent kernel $\mathcal{R}\left(\frac{1}{2}+i t ; z, w\right)$ is defined for $\operatorname{Im} t<0$ by (3.3) and for $t \in \mathbb{R} \backslash\{0\}$ by (3.7) with $t= \pm\left(\mu_{0}-\frac{1}{4}\right)^{\frac{1}{2}}$. For $\operatorname{Im} t>0$ we define $\mathcal{R}\left(\frac{1}{2}+i t ; z, w\right)$ 
by

$$
\mathcal{R}\left(\frac{1}{2}+i t ; z, w\right)=\mathcal{R}\left(\frac{1}{2}-i t ; z, w\right)+i t^{-1} E_{j}\left(z, \frac{1}{2}+i t\right) \bar{E}_{j}\left(w, \frac{1}{2}+i t\right)
$$

where by $(2.14)$

$$
\left\{E_{j}\left(z, \frac{1}{2}+i t\right)\right\}_{j=1}^{m}=C\left(\frac{1}{2}+i t\right)\left\{E_{j}\left(z, \frac{1}{2}-i t\right)\right\}_{j=1}^{m}
$$

By (3.9) and Theorem 2.3, $\mathcal{R}\left(\frac{1}{2}+i t ; z, w\right)$ as given by (3.10) and (3.11) defines an analytic continuation of the resolvent kernel from $\mathbb{C}^{-}$across $\mathbb{R} \backslash\{0\}$ to $\mathbb{C}^{+}$.

Since $E_{j}\left(z, \frac{1}{2}+i t\right) \sim y_{j}^{\frac{1}{2}+\tau}$ for $y_{j} \rightarrow \infty$ in the cusp $Z_{j}$, where $\tau=\operatorname{Im} t>0$, the kernel $\mathcal{R}\left(\frac{1}{2}+i t ; z, w\right)$ defines an operator $\tilde{R}\left(\frac{1}{2}+i t\right)$ in $\mathcal{B}\left(\left(\mathrm{L}_{(\tau+\delta)}^{2}(M)\right), \mathrm{L}_{-(\tau+\delta)}^{2}(M)\right)$ for every $\delta>0$.

We show in Appendix 3 that

$$
\left\|R\left(\frac{1}{2}+i t\right)\right\|_{\mathcal{B}\left(\mathrm{L}_{\delta}^{2}, \mathrm{~L}_{-\delta}^{2}\right)}<C \quad \text { for } \operatorname{Im} t<0,|t| \geq \kappa>0 .
$$

This together with the weak analyticity of $R\left(\frac{1}{2}+i t\right)$ in $|\operatorname{Im} t|<\delta$ implies that $\tilde{R}\left(\frac{1}{2}+i t\right)$ is analytic in $\{t|| \operatorname{Im} t \mid<\delta\} \backslash\{0\}$ as a function with values in $\mathcal{B}\left(\mathrm{L}_{\delta}^{2}(M), \mathrm{L}_{-\delta}^{2}(M)\right)$.

By (3.10) and (3.11), the set of poles of $\tilde{R}\left(\frac{1}{2}+i t\right) \in \mathcal{B}\left(\mathrm{L}_{\delta}^{2}(M), \mathrm{L}_{-\delta}^{2}(M)\right)$ is precisely the set of poles of $C\left(\frac{1}{2}+i t\right)$ in $\{t \mid 0<\operatorname{Im} t<\delta\}$. (We are restricting to $\mathcal{H}_{c}(\Delta)$ and hence omitting $\left.\sigma_{d}(\Delta)\right)$.

From this section and the results of section 2 we obtain

Theorem 3.1. In the strip $S_{\delta}=\left\{s \mid \frac{1}{2}-\delta<\operatorname{Re} s<\frac{1}{2}\right\}$, where $0<\delta \leqq \frac{1}{2}$, the poles of $C(s)$, the poles of $\tilde{R}(s) \in \mathcal{B}\left(\mathrm{L}_{\delta}^{2}(M), \mathrm{L}_{-\delta}^{2}(M)\right)$ and the resolvent resonances $\bigcup_{0<\operatorname{Arg} \lambda<\pi}\left\{\sigma_{d}(\lambda) \cap S_{\delta}\right\}$ coincide.

Proof. We have seen above that the poles of $C(s)$ and $\tilde{R}(S)$ coincide. A point $\rho$ is a resolvent resonance in $S_{\delta}$ iff for some $f, g$ in the dense set $\mathcal{F}$ of analytic vectors the analytic continuation of $(f, R(s) g)$ across $\frac{1}{2}+i \mathbb{R}$ to $S_{\delta}$ has a pole at $\rho$. 
Since all functions in $\mathcal{F}$ lie in $\mathrm{L}_{\delta}^{2}(\mu)$ for $\delta<\frac{1}{2}$, it follows that this holds iff $\rho$ is a pole of the analytically continued resolvent $\tilde{R}(s) \in \mathcal{B}\left(\mathrm{L}_{\delta}^{2}(M), \mathrm{L}_{-\delta}^{2}(M)\right)$.

\section{Resonances and resonance functions.}

Let $\rho$ be a pole of $C(s)$. Then $\rho$ is also a pole of $E(z, s)$, and $\Phi_{\rho k}(z)=$ $\operatorname{Res}_{\rho} E_{k}(z, s)$ is a solution of

$$
\Delta \Phi_{\rho k}(z)=\rho(1-\rho) \Phi_{\rho k}(z)
$$

The functions $\Phi_{\rho k}(z), k=1, \cdots, m$ are called the resonance functions corresponding to $\rho$. By (2.1), $\Phi_{\rho k}(z)$ has for $z \in Z_{j}, y_{j}>a_{j}$, the Fourier expansion

$$
\left\{\Phi_{\rho k}(z)\right\}_{k=1}^{m}=\operatorname{Res}_{\rho} C(s)\left\{y_{j}^{1-\rho}\right\}_{j=1}^{m}+\operatorname{Res}_{\rho} \sum_{l}^{\prime}
$$

where $\operatorname{Res}_{\rho} \sum_{l}^{\prime}=O\left(e^{-c y_{j}}\right)$ as $y_{j} \rightarrow \infty$ and $U_{\lambda}\left(\operatorname{Res}_{\rho} \sum_{l}^{\prime}\right)=\operatorname{Res}_{\rho} \sum_{l}^{\prime}$.

The components $y_{j}^{1-\rho}$ of the leading terms transform under $U_{\lambda}^{0 j}$ into $U_{\lambda}^{0 j} y_{j}^{1-\rho}$, which for $y_{j}>R_{j}$ are given according to (2.17) by

$$
U_{\lambda}^{0 j} y_{j}^{1-\rho}=\lambda^{\frac{1}{2}} y_{j}^{\frac{1}{2}-\lambda\left(\rho-\frac{1}{2}\right)}=\lambda^{\frac{1}{2}} y_{j}^{\frac{1}{2}-i \lambda \mu}, \rho-\frac{1}{2}=i \mu .
$$

For $0 \geq \operatorname{Arg} \lambda \geq-\operatorname{Arg} \mu$, the function $U_{\lambda}^{0 j} y_{j}^{1-\rho}$ and hence $\Phi_{\rho k}(\lambda)=$ $U_{\lambda} \Phi_{\rho k}$ are not in $L^{2}(M)$. For $-\operatorname{Arg} \mu>\operatorname{Arg} \lambda>-\pi$, the functions $\Phi_{\rho k}(\lambda), k=1, \cdots, m$, are in $\mathrm{L}^{2}(M)$, so they are eigenfunctions of $\Delta(\lambda)$ with eigenvalue $\rho(1-\rho)$.

As $\operatorname{Arg} \lambda \rightarrow-\pi$, all poles of $C(s)$ become eigenvalues of some $\Delta(\lambda)$. By Theorem 2.3, the poles of $C(s)$ are symmetric with respect to the $x$-axis.

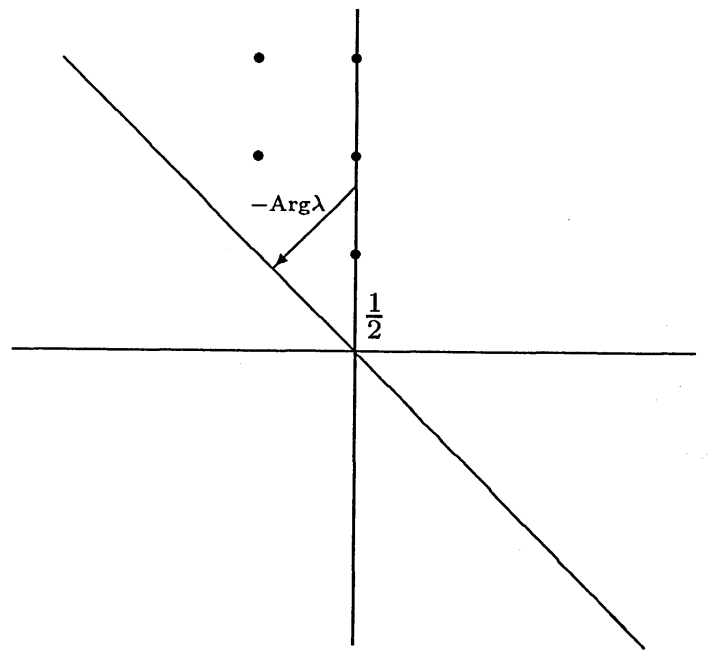


If $\lambda=e^{i \varphi}, \mu=\left|\rho-\frac{1}{2}\right| e^{i \eta}$, the modulus of $\Phi_{\rho k}(\lambda)$ in $Z_{k}$ approaches $c y_{k}^{\frac{1}{2}-\left|\rho-\frac{1}{2}\right| \sin \eta}=c y^{\operatorname{Re} \rho}$ as $\varphi \rightarrow-\pi$.

We have proved

Theorem 4.1. Let $\rho$ be a pole of $C(s)$ with resonance functions $\Phi_{\rho k}, k=$ $1, \ldots, m$. Then the functions $\Phi_{\rho k}(\lambda)$ defined for $\lambda \in(1-\alpha, \infty)$ by $\Phi_{\rho k}(\lambda)=$ $U_{\lambda} \Phi_{\rho k}$ have analytic extensions to $\lambda \in G_{\alpha}$, and for $k=1, \ldots, m$ and $\lambda \in G_{\alpha}$

$$
\Delta(\lambda) \Phi_{\rho k}(z, \lambda)=\rho(1-\rho) \Phi_{\rho k}(z, \lambda)
$$

For $-\operatorname{Arg} \eta>\operatorname{Arg} \lambda>-\pi, \Phi_{\rho k}(\lambda)$ is an eigenfunction of $\Delta(\lambda)$ where $\rho=$ $\frac{1}{2}+i \eta$.

For $\operatorname{Arg} \lambda \rightarrow-\pi,\left|\Phi_{\rho k}(z, \lambda)\right| \rightarrow c y_{k}^{\mathrm{Re} \rho}$ for $z \in Z_{k}$. The poles of $C(s)$ are symmetric with respect to the $x$-axis.

We finally discuss the discrete eigenvalues $\mu_{j}, j=1, \ldots, k$, of $\Delta$. These eigenvalues either correspond to cusp forms, in which case they remain eigenvalues of $\Delta(\lambda)$ for all $\lambda \in G_{\alpha}$, or the associated eigenfunctions $u_{j k}$ are not cusp forms, in which case the $\mu_{j}$ are poles of $C(s)$ and $E(s, z)$ and we can carry out a similar analysis to the one given above for resonance functions. We obtain for the leading term of $u_{j k}$ in the $k$-th cusp $Z_{k}$

$$
U_{\lambda}^{0 k} y^{1-\mu_{k}}=\lambda^{1 / 2} y_{k}^{\frac{1}{2}+\left(\frac{1}{2}-\mu_{k}\right) \lambda}
$$

This implies

Theorem 4.2. Let $\mu_{j} \in\left(\frac{1}{2}, 1\right)$ be a discrete eigenvalue of $\Delta$ with eigenfunctions $u_{j k}, k=1, \ldots, m$. Then the functions $u_{j l}(z, \lambda), j=1, \ldots, k, l=$ $1, \ldots, m$, defined for $\lambda \in(1-\alpha, \infty)$ by

$$
u_{j l}(z, \lambda)=U_{\lambda} u_{j l}(z)
$$

have analytic extensions to $\lambda \in G_{\alpha}$, and for $\lambda \in G_{\alpha}$

$$
\Delta(\lambda) u_{j l}(z, \lambda)=\mu_{j}\left(1-\mu_{j}\right) u_{j l}(z, \lambda)
$$

For $|\operatorname{Arg}|<\frac{\pi}{2}, u_{j l}(\bullet, \lambda)$ is an eigenfunction of $\Delta(\lambda)$.

If $u_{j l}$ is a cusp form, then $u_{j l}(z, \lambda)$ is independent of $\lambda$ and hence an eigenfunction of $\Delta(\lambda)$ for $|\operatorname{Arg} \lambda|<\pi$. 


\section{Analytic Perturbation of $\Delta$ and Fermi's golden rule.}

We now specialize to hyperbolic manifolds $M$ of constant curvature. In this case $M$ can be represented as a quotient $\Gamma \backslash h$, where $h$ is the upper half-plane with the Poincare metric $d s^{2}=y^{-2}\left(d x^{2}+d y^{2}\right)$ and $\Gamma$ a discrete subgroup of $\mathrm{P}-\mathrm{SL}_{2}(\mathbb{R})$. We consider a deformation of $M$ in the Teichmüller space $T(\Gamma)$, following Phillips and Sarnak [P-S1]. A tangent vector of $T(\Gamma)$ at $\Gamma$ is given by a holomorphic cusp form $Q$ of weight 4 for $\Gamma$, i.e. $Q$ is a holomorphic function on $h$ with Fourier expansion in each cusp of the form

$$
Q(z)=\sum_{n=1}^{\infty} a_{n} e^{2 \pi i n z}
$$

and satisfying $Q(\gamma z)=(c z+d)^{4} Q(z)$ for $\left(\begin{array}{ll}a & b \\ c & d\end{array}\right) \in \Gamma$ and $z \in h$. Let $\hat{g}(z, \varepsilon)$ be the metric with line element

$$
d s^{2}=g_{i j}(z, \varepsilon) d x^{i} d x^{j}=y^{-2}\left|d z+\varepsilon y^{2} \overline{Q(z)} d \bar{z}\right|^{2}
$$

with $|\varepsilon|<\varepsilon_{0}=\left\|y^{2} Q\right\|_{\infty}^{-1}$.

We denote by $\Delta_{Q}(\varepsilon)$ the Laplacian of $M$ with the metric $\hat{g}(z, \varepsilon) . \Delta_{Q}(\varepsilon)$ is given by $\Delta_{Q}(\varepsilon)=\Delta+\varepsilon \mathrm{L}_{Q}+O\left(\varepsilon^{2}\right)$, where $\mathrm{L}_{Q}=-8 R e\left(Q\left(y^{2} \frac{\partial}{\partial \bar{z}}\right)^{2}\right)$. We utilize the following result proved in [P-S1] as lemma 2.2:

Lemma 5.1 (Phillips and Sarnak). There exist smooth diffeomorphisms $\phi_{\varepsilon}$ of $M$ onto $M$, depending on b, such that in each cusp neighbourhood $Z_{j}$

(i) $\phi_{\varepsilon}^{*}(\hat{g}(\varepsilon))=\hat{g}(0)$ for $y_{j}>2 b$.

(ii) $\phi_{\varepsilon}^{*}(\hat{g}(\varepsilon))=\hat{g}(\varepsilon)$ for $y_{j}<b$.

(iii) $\phi_{0}=\mathrm{Id} ; \phi_{\varepsilon}$ is real-analytic in $\varepsilon$ and varies smoothly in $z$ and $\varepsilon$, and $\left|\frac{\partial \phi_{\varepsilon}}{\partial \varepsilon}\right|=O(1)$ uniformly for $b<y_{j}<2 b$

Let $\tilde{g}(\varepsilon)=\phi_{\varepsilon}^{*}(\hat{g}(\varepsilon)),-\varepsilon_{0}<\varepsilon<\varepsilon_{0}$. Then $\phi_{\varepsilon}$ is an isometry of $\left.M, \hat{g}(\varepsilon)\right)$ onto $(M, \tilde{g}(\varepsilon))$. Let $\tilde{\Delta}_{Q}(\varepsilon)$ denote the Laplacian on $M$ with respect to $\tilde{g}(\varepsilon)$. Then $\tilde{\Delta}_{Q}(\varepsilon)=\Delta$ for $y>2 b$. Since $\tilde{\Delta}_{Q}(0)=\Delta$, we have an expansion of the form

$$
\tilde{\Delta}_{Q}(\varepsilon)=\Delta+\varepsilon \tilde{\mathrm{L}}_{Q}+O\left(\varepsilon^{2}\right), \varepsilon \in\left(-\varepsilon_{0}, \varepsilon_{0}\right)
$$


The operators $\tilde{\Delta}_{Q}(\varepsilon)$ act for each $\varepsilon$ on a manifold $M$ with a different metric $\tilde{g}(\varepsilon)$. For the purpose of applying analytic perturbation theory we map as in [P-S1] $\mathrm{L}^{2}(M ; \tilde{g}(\varepsilon))$ isometrically to the fixed Hilbert space $\mathrm{L}^{2}(M ; \tilde{g}(0))$ by the transformations $\tau_{\varepsilon}$ defined as multiplication by the function $d_{\varepsilon}^{1 / 2}$, where

$$
d_{\varepsilon}=\operatorname{det} \tilde{g}(\varepsilon) / \operatorname{det} \hat{g}(0)
$$

Since $\tilde{g}(\varepsilon)$ differs from $\hat{g}(0)$ only on a compact set, $J_{\varepsilon}$ is a real-analytic family of operators. Then $\left\{\tilde{\tilde{\Delta}}_{Q}(\varepsilon)\right\}$ defined by

$$
\tilde{\tilde{\Delta}}_{Q}(\varepsilon)=\tau_{\varepsilon} \tilde{\Delta}_{Q}(\varepsilon) \tau_{\varepsilon}^{-1}
$$

is a real-analytic, self-adjoint family of operators in the Hilbert space $\mathrm{L}^{2}(M ; \hat{g}(0))$ and $\tilde{\tilde{\Delta}}_{Q}(0)=\Delta$.

We calculate the first order coefficient $\tilde{\tilde{\mathrm{L}}}_{Q}$ of $\tilde{\tilde{\Delta}}_{Q}(\varepsilon)$ in the expansion around $\varepsilon=0$. Inserting

$$
d_{\varepsilon}=1+d^{\prime}(0) \varepsilon+O\left(\varepsilon^{2}\right)
$$

and (5.1) in (5.2), we obtain

$$
\begin{aligned}
\tilde{\tilde{\Delta}}_{Q}(\varepsilon) & =\left(1+\frac{1}{2} d^{\prime}(0) \varepsilon+O\left(\varepsilon^{2}\right)\right)\left(\Delta+\varepsilon \tilde{\mathrm{L}}_{Q}+O\left(\varepsilon^{2}\right)\right)\left(1-\frac{1}{2} d^{\prime}(0) \varepsilon+O\left(\varepsilon^{2}\right)\right) \\
& =\Delta+\varepsilon\left(\tilde{\mathrm{L}}_{Q} \frac{1}{2}\left(d^{\prime}(0) \Delta-\Delta d^{\prime}(0)\right)\right)+O\left(\varepsilon^{2}\right)
\end{aligned}
$$

Thus, $\tilde{\tilde{\mathrm{L}}}_{Q}=\tilde{\mathrm{L}}_{Q}+\frac{1}{2}\left(d^{\prime}(0) \Delta-\Delta d^{\prime}(0)\right)$. Note that $\tilde{\tilde{\mathrm{L}}}_{Q}=0$ for $y>2 b$.

For each $\varepsilon \in\left(-\varepsilon_{0}, \varepsilon_{0}\right)$ we apply the complex power $U_{\lambda}$ of section 1 to $\tilde{\Delta}_{Q}(\varepsilon)$ with $K>2 b$ and obtain

$$
\tilde{\Delta}_{Q}(\varepsilon, \lambda)=\Delta(\lambda)+\varepsilon \tilde{\mathrm{L}}_{Q}(\lambda)+O_{\lambda}\left(\varepsilon^{2}\right)
$$

where $\tilde{\Delta}_{Q}(\varepsilon, \lambda)$ and $\tilde{\mathrm{L}}_{Q}(\lambda)$ are the analytic extensions of $U_{\lambda} \tilde{\Delta}_{Q}(\varepsilon) U_{\lambda}^{-1}$ and $U_{\lambda} \tilde{\mathrm{L}}_{Q} U_{\lambda}^{-1}$ resp. from $\lambda \in(1-\alpha, \infty)$ to $\lambda \in G_{\alpha}$.

Since

$$
\left(U_{\lambda} f\right)\left(x_{j}, y_{j}\right)=f\left(x_{j}, y_{j}\right) \text { for } y_{j} \leq 2 b, j=1, \ldots, m,
$$

we have

$$
\tilde{\Delta}_{Q}(\varepsilon, \lambda)=\tilde{\Delta}_{Q}(\varepsilon) \text { for } y_{j} \leq 2 b, j=1, \ldots, m
$$


Moreover,

$$
\tilde{\Delta}_{Q}(\varepsilon)=\Delta \text { for } y_{j}>2 b, j=1, \ldots, m
$$

and hence

$$
\tilde{\Delta}_{Q}(\varepsilon, \lambda)=\Delta(\lambda) \text { for } y_{j}>2 b, j=1, \ldots, m .
$$

Thus $\tilde{\mathrm{L}}_{Q}(\lambda)=\tilde{\mathrm{L}}_{Q}$ and $O_{\lambda}\left(\varepsilon^{2}\right)$ are independent of $\lambda \in G_{\alpha}$, so that

$$
\tilde{\Delta}_{Q}(\varepsilon, \lambda)=\Delta(\lambda)+\varepsilon \tilde{\mathrm{L}}_{Q}+O\left(\varepsilon^{2}\right) .
$$

Applying the family of transformations $\left\{\tau_{\varepsilon}\right\}$ as in $(5.2)$ to $\tilde{\Delta}_{Q}(\varepsilon, \lambda)$, we obtain

$$
\tilde{\tilde{\Delta}}_{Q}(\varepsilon, \lambda)=\tau_{\varepsilon} \tilde{\Delta}_{Q}(\varepsilon) \tau_{\varepsilon}^{-1}
$$

and as in (5.3)

$$
\tilde{\tilde{\Delta}}_{Q}(\varepsilon, \lambda)=\Delta(\lambda)+\varepsilon\left(\tilde{\mathrm{L}}_{Q}+\frac{1}{2}\left(d^{\prime}(0) \Delta(\lambda)-\Delta(\lambda) d^{\prime}(0)\right)\right)+O\left(\varepsilon^{2}\right)
$$

with the first order perturbation

$$
\tilde{\tilde{\mathrm{L}}}_{Q}(\lambda)=\tilde{\mathrm{L}}_{Q}+\frac{1}{2}\left(d^{\prime}(0) \Delta(\lambda)-\Delta(\lambda) d^{\prime}(0)\right)
$$

Note that $\tilde{\tilde{L}}_{Q}$ and the $O\left(\varepsilon^{2}\right)$-term are 0 for $y_{j}>2 b$, so $\tilde{\tilde{\Delta}}_{Q}(\varepsilon, \lambda)=\Delta(\lambda)$ for $y_{j}>2 b, j=1, \ldots, m, \varepsilon \in\left(-\varepsilon_{0}, \varepsilon_{0}\right)$.

The maps $\phi_{\varepsilon}$ and $\tau_{\varepsilon}$ are isometries and hence induce unitary operators on the corresponding $\mathrm{L}^{2}$-spaces:

$$
\mathrm{L}^{2}(M ; \hat{g}(\varepsilon)) \stackrel{\phi_{\varepsilon}}{\rightarrow} \mathrm{L}^{2}(M ; \tilde{g}(\varepsilon)) \stackrel{\tau_{\varepsilon}}{\rightarrow} \mathrm{L}^{2}(M ; \hat{g}(0)) .
$$

Hence the spectra of $\Delta_{Q}(\varepsilon, \lambda), \tilde{\Delta}_{Q}(\varepsilon, \lambda)$ and $\tilde{\tilde{\Delta}}_{Q}(\varepsilon, \lambda)$ are identical. We can therefore study the variation of the discrete eigenvalues of $\Delta_{Q}(\varepsilon, \lambda)$ with $\varepsilon$ by studying it for $\tilde{\tilde{\Delta}}_{Q}(\varepsilon, \lambda)$. We denote by $\sigma_{e}(\varepsilon, \lambda)$ and $\sigma_{d}(\varepsilon, \lambda)$ the essential and discrete spectrum of $\Delta_{Q}(\varepsilon, \lambda)$ resp.

$$
\begin{gathered}
\text { Since } \tilde{\tilde{\Delta}}_{Q}(\varepsilon, \lambda)=\Delta_{Q}(\lambda) \text { for } y_{j}>2 b, j=1, \ldots, m, \text { and } \varepsilon \in\left(-\varepsilon_{0}, \varepsilon_{0}\right), \\
\sigma_{e}(\varepsilon, \lambda)=\lambda^{-2} \overline{\mathbb{R}^{+}}+\frac{1}{4} \text { for all } \varepsilon \in\left(-\varepsilon_{0}, \varepsilon_{0}\right) .
\end{gathered}
$$

Theorem 1.1 applies to $\tilde{\tilde{\Delta}}_{Q}(\varepsilon, \lambda)$ for each $\varepsilon \in\left(-\varepsilon_{0}, \varepsilon_{0}\right)$. We reformulate this explicitly, since this is the basis for the main perturbation result, Fermi's golden rule. 
Theorem 5.2. $\left\{\tilde{\tilde{\Delta}}_{Q}(\varepsilon, \lambda)\right\}_{\lambda \in G_{\alpha}}$ is for each $\varepsilon \in\left(-\varepsilon_{0}, \varepsilon_{0}\right)$ a self-adjoint, analytic family of type $A$ in $\mathrm{L}^{2}(M ; \hat{g}(0))$ with domain $\mathcal{D}(\Delta)$.

For each $\lambda \in G_{\alpha}, \tilde{\tilde{\Delta}}_{Q}(\varepsilon, \lambda)$ is real-analytic in $\varepsilon$ for $\varepsilon \in\left(-\varepsilon_{0}, \varepsilon_{0}\right)$.

$$
\begin{gathered}
\sigma_{e}(\varepsilon, \lambda)=\lambda^{-2} \overline{\mathbb{R}^{+}}+\frac{1}{4} \text { for } \varepsilon \in\left(-\varepsilon_{0}, \varepsilon_{0}\right), \lambda \in G_{\alpha} \\
\sigma_{d}(\varepsilon, \lambda) \cap\left(\frac{1}{4}, \infty\right)=\sigma_{p}\left(\tilde{\tilde{\Delta}}_{Q}(\varepsilon)\right)
\end{gathered}
$$

For $\operatorname{Re} \lambda>0, \lambda \notin(0,1-\alpha]$

$$
\sigma_{d}(\varepsilon, \lambda) \cap\left[0, \frac{1}{4}\right)=\sigma_{d}\left(\tilde{\tilde{\Delta}}_{Q}(\varepsilon)\right)
$$

For all $\varepsilon \in\left(-\varepsilon_{0}, \varepsilon_{0}\right), \sigma_{d}(\varepsilon, \lambda) \backslash \mathbb{R}$ is contained in the angular region between $\frac{1}{4}+\overline{\mathbb{R}^{+}}$and $\frac{1}{4} \lambda^{-2} \overline{\mathbb{R}^{+}}$and otherwise independent of $\lambda$.

From theorem 5.2 and Kato's analytic perturbation theory $[\mathrm{K}]$ the proof of Fermi's Golden Rule is straightforward. This was observed and proved by $\mathrm{B}$. Simon [Si] in the case of Schrödinger operators, where the analogue of Theorem 5.2 was provided by the dilation-analytic theory [B-C]. For the sake of completeness we include the proof according to [Si], adapting it to the case of general real-analytic perturbations and embedded eigenvalues of finite multiplicity, a commonly occurring case for the Laplacian on hyperbolic manifolds.

Theorem 5.3. Let $\kappa>\frac{1}{4}$ be an embedded eigenvalue of $\Delta$ of multiplicity $k \geq 1$, and let $P_{0}$ be the orthogonal projection on $\operatorname{Ker}(\Delta-\kappa)$.

Let $\tilde{\tilde{\Delta}}_{Q}(\varepsilon, \lambda)=\Delta+\varepsilon \tilde{\tilde{\mathrm{L}}}_{Q}(\lambda)+O\left(\varepsilon^{2}\right)$ as in (5.3). For fixed $\lambda \in G_{\alpha}$ with $-\frac{\pi}{2}<\operatorname{Arg} \lambda<0$, let $\hat{\kappa}(\varepsilon)=\frac{1}{k} \sum_{j=1}^{k} \kappa_{j}(\varepsilon)$ be the average of the $\kappa$-group of eigenvalues of $\tilde{\tilde{\Delta}}(\varepsilon, \lambda)$, which arise from the splitting of $\kappa$ when $\varepsilon \neq o$. Then $\hat{\kappa}(\varepsilon)$ is real-analytic for $\varepsilon \in\left(-\varepsilon_{1}, \varepsilon_{1}\right)$, where $\varepsilon_{1} \leq \varepsilon_{0}$ is chosen such that $\kappa_{j}(\varepsilon)$ remain discrete eigenvalues of $\tilde{\tilde{\Delta}}_{Q}(\varepsilon, \lambda)$ for $j=1, \ldots, k$. Let

$$
\hat{\kappa}(\varepsilon)=\kappa+\hat{a}_{1} \varepsilon+\hat{a}_{2} \varepsilon^{2}+O\left(\varepsilon^{2}\right)
$$

be the expansion of $\hat{\kappa}(\varepsilon)$ around $\varepsilon=0$. Then $\hat{a}_{1}$ is real, and $\operatorname{Im} \hat{a}_{2}$ is given by

$$
\operatorname{Im} \hat{a}_{2}=\pi \frac{d}{d \mu}\left[\frac{1}{k} \sum_{j=1}^{k}\left(\tilde{\tilde{\mathrm{L}}}_{Q} u_{j}, P^{\prime}(\kappa-\delta, \mu) \tilde{\tilde{\mathrm{L}}}_{Q} u_{j}\right)\right]_{\mu=\kappa}
$$


where

$$
P^{\prime}(\mu-\delta, \mu)= \begin{cases}P((\kappa-\delta, \mu]) & \text { for } \kappa-\delta<\mu<\kappa \\ P((\kappa-\delta, \mu]) \ominus P_{0} & \text { for } \mu \geq \mu_{2} \kappa\end{cases}
$$

and $P((\kappa-\delta, \mu])$ is the spectral projection of $\Delta$ corresponding to the interval $(\kappa-\delta, \mu], \delta>0$, while $\left\{u_{1}, \ldots, u_{k}\right\}$ is an orthonormal basis of $\operatorname{ker}(\Delta-\kappa)$. The function $f(\mu)$ under the differentiation sign on the r.h.s. of (5.7) is $C^{\infty}(\kappa-\delta, \kappa,+\delta)$. Here $\delta>0$ is chosen such that the interval $(\kappa-\delta, \kappa,+\delta)$ contains no other eigenvalues of $\Delta$.

Proof. $\hat{a}_{1}$ is a real, since otherwise $\hat{\kappa}(\varepsilon)$ and hence at least one $\kappa_{j}(\varepsilon)$ would have negative imaginary part for some $\varepsilon$ with $|\varepsilon|<\varepsilon_{1}$, in contradiction to the fact that $\kappa_{j}(\varepsilon)$ lies in the angle between $\frac{1}{4}+\overline{\mathbb{R}^{+}}$and $\frac{1}{4}+(1+\lambda)^{-2} \overline{\mathbb{R}^{+}}$ for $\operatorname{Arg} \lambda<0,|\varepsilon|<\varepsilon_{1}$. We have

$$
\begin{array}{r}
f(\mu)=\frac{1}{k} \sum_{j=1}^{k} \lim _{\nu \downarrow 0} \int_{\kappa-\delta}^{\mu} d t \operatorname{Im}\left[\frac{1}{\pi}\left(\tilde{\tilde{\mathrm{L}}}_{Q} u_{j},(\Delta-t-i \nu)^{-1} \tilde{\tilde{\mathrm{L}}}_{Q} u_{j}\right)\right. \\
\left.-\frac{1}{\pi} \frac{\left(\tilde{\tilde{\mathrm{L}}}_{Q} u_{j}, P_{0} \tilde{\mathrm{L}}_{Q} u_{j}\right)}{\kappa-t-i \nu}\right]
\end{array}
$$

We now apply a standard dilation-analytic argument. For $\lambda \in(1-\alpha, \infty)$,

$$
\begin{aligned}
& \left(\tilde{\tilde{\mathrm{L}}}_{Q} u_{j},(\Delta-t-i \nu)^{-1} \tilde{\tilde{\mathrm{L}}}_{Q} u_{j}\right)-\frac{\left(\tilde{\tilde{\mathrm{L}}}_{Q} u_{j}, P_{0} \tilde{\tilde{\mathrm{L}}}_{Q} u_{j}\right)}{\kappa-t-i \nu}= \\
& \left(\tilde{\tilde{\mathrm{L}}}_{Q}(\bar{\lambda}) u_{j},(\Delta(\lambda)-t-i \nu)^{-1} \tilde{\tilde{\mathrm{L}}}_{Q}(\lambda) u_{j}\right)-\frac{\left(\tilde{\tilde{\mathrm{L}}}_{Q}(\bar{\lambda}) u_{j}, P_{0} \tilde{\tilde{\mathrm{L}}}_{Q}(\lambda) u_{j}\right)}{k-t-i \nu}
\end{aligned}
$$

By 4) of Theorem 1.1, $t+i \nu \notin \sigma(\Delta(\lambda))$ for $\operatorname{Arg} \lambda>-\frac{1}{2} \operatorname{Arg}(t+i \nu)$, hence the r.h.s. of (5.8) is analytic in $\lambda$ for such $\lambda$. Since it is constant for $\lambda \in(1-\alpha, \infty)$, it follows that it is constant for all $\lambda \in G_{\alpha}$ with $\operatorname{Arg} \lambda>$ $-\frac{1}{2} \operatorname{Arg}(t+i \nu)$, i.e. (5.8) holds for all such $\lambda$. Hence (5.8) holds for $\nu>0$ and $\operatorname{Arg} \lambda>0$. Fixing $\lambda$ with $\pi>\operatorname{Arg} \lambda>0$, we can now let $\nu \downarrow 0$ and 
obtain for $0<|t-\kappa|<\delta$.

$$
\begin{aligned}
& \lim _{\nu \downarrow 0}\left[\frac{1}{\pi}\left(\tilde{\tilde{\mathrm{L}}}_{Q} u_{j},(\Delta-t-i \nu)^{-1} \tilde{\mathrm{L}}_{Q} u_{j}\right)-\frac{1}{\pi} \frac{\left(\tilde{\tilde{\mathrm{L}}}_{Q} u_{j}, P_{0} \tilde{\tilde{\mathrm{L}}}_{Q} u_{j}\right)}{\kappa-t-i \nu}\right]= \\
& \frac{1}{\pi}\left(\tilde{\tilde{\mathrm{L}}}_{Q}(\bar{\lambda}) u_{j},(\Delta(\lambda)-t)^{-1} \tilde{\tilde{\mathrm{L}}}_{Q}(\lambda) u_{j}\right)-\frac{1}{\pi} \frac{\left(\tilde{\tilde{\mathrm{L}}}_{Q}(\bar{\lambda}) u_{j}, P_{0} \tilde{\tilde{\mathrm{L}}}_{Q}(\lambda) u_{j}\right)}{k-t}
\end{aligned}
$$

which has a removable singularity at $t=\kappa$. Hence $f \in C^{\infty}(\kappa-\delta, \kappa+\delta)$.

We now fix $\lambda$ with $\pi>\operatorname{Arg} \lambda>0$. Then $\kappa$ is a discrete, semisimple eigenvalue, i.e. a simple pole of $(\Delta(\lambda)-\zeta)^{-1}$, and $\kappa_{j}(\varepsilon)$ a discrete eigenvalue of $\tilde{\tilde{\Delta}}(\varepsilon, \lambda)$ for $|\varepsilon|<\varepsilon_{1}, j=1, \ldots k$.

By [K] II $\S 2(2.33)(\mathrm{p} 79), \hat{\kappa}(\varepsilon)=\frac{1}{m} \sum_{j=1}^{k} \kappa_{j}(\varepsilon)$ is real-analytic in $\varepsilon$, and

$$
\hat{a}_{2}=\frac{1}{k} \operatorname{tr}\left(\tilde{\tilde{\mathrm{L}}}_{Q 2}(\lambda) P_{0}-\tilde{\tilde{\mathrm{L}}}_{Q}(\lambda) S(\lambda) \tilde{\tilde{\mathrm{L}}}_{Q}(\lambda) P_{0}\right)
$$

where

$$
\tilde{\tilde{\Delta}}_{Q}(\varepsilon, \lambda)=\Delta+\tilde{\tilde{\mathrm{L}}}_{Q} \varepsilon+\tilde{\tilde{\mathrm{L}}}_{Q 2} \varepsilon^{2}+O\left(\varepsilon^{3}\right)
$$

and $S(\lambda)$ is the reduced resolvent of $\Delta(\lambda)$, given by

$$
(\Delta(\lambda)-(\kappa+i \nu))^{-1}=\frac{P_{0}}{-i \nu}+S(\lambda)+\sum_{k=1}^{\infty}(-i \nu)^{k} A_{k}(\lambda)
$$

Thus, for small $\delta>0$

$$
\begin{aligned}
& \hat{a}_{2}=\frac{1}{k}\left[\operatorname{tr}\left(\tilde{\tilde{\mathrm{L}}}_{Q 2}(\varepsilon) P_{0}\right)\right. \\
&\left.\frac{1}{2 \pi i} \sum_{j=1}^{k} \int_{|\mu-\kappa|=\delta} \frac{d \mu}{\kappa-\mu}\left(u_{j}, \tilde{\tilde{\mathrm{L}}}_{Q}(\Delta(\lambda)-\mu)^{-1} \tilde{\tilde{\mathrm{L}}}_{Q} u_{j}\right)\right]
\end{aligned}
$$

The function $g_{j}(\mu)=\left(u_{j}, \tilde{\tilde{\mathrm{L}}}_{Q}(\Delta(\lambda)-\mu)^{-1} \tilde{\tilde{\mathrm{L}}}_{Q} u_{j}\right)$ has a pole term

$$
\left(u_{j}, \tilde{\mathrm{L}}_{Q} P_{0} \tilde{\tilde{\mathrm{L}}}_{Q} u_{j}\right) \frac{1}{\kappa-\mu}
$$

at $\mu=\kappa$, hence by the complex transformation argument

$$
\begin{aligned}
\hat{a}_{2}= & \frac{1}{k} \operatorname{tr}\left(\tilde{\tilde{\mathrm{L}}}_{Q 2}(\lambda) P_{0}\right)+\frac{1}{k} \sum \operatorname{Res}\left(\frac{g_{j}(\mu)}{\kappa-\mu}\right)_{\mu=\kappa} \\
=\frac{1}{k} \operatorname{tr}\left(\tilde{\tilde{\mathrm{L}}}_{Q 2}(\lambda) P_{0}\right) & \\
& \quad+\frac{1}{k} \sum_{j=1}^{k} \lim _{\mu \rightarrow \kappa}\left[\left(u_{j}, \tilde{\tilde{\mathrm{L}}}_{Q}(\Delta-\mu)^{-1} \tilde{\tilde{\mathrm{L}}}_{Q} u_{j}\right)-\left(u_{j}, \tilde{\tilde{\mathrm{L}}}_{Q} P_{0} \tilde{\tilde{\mathrm{L}}}_{Q} u_{j} \frac{1}{\kappa-\mu}\right)\right]
\end{aligned}
$$


Note that

$$
\operatorname{tr}\left(\tilde{\tilde{\mathrm{L}}}_{Q 2}(\lambda) P_{0}\right)=\sum_{j=1}^{k}\left(u_{j}, \tilde{\tilde{\mathrm{L}}}_{Q 2}(\lambda) u_{j}\right)
$$

is real. Hence, letting $\nu=\kappa+i \nu$

$$
\begin{aligned}
& \operatorname{Im} \hat{a}_{2}= \lim _{\nu \downarrow 0} \frac{1}{k} \sum_{j=1}^{k} \operatorname{Im}\left[\left(u_{j}, \tilde{\tilde{\mathrm{L}}}_{Q}(\Delta-\kappa-i \nu)^{-1} \tilde{\tilde{\mathrm{L}}}_{Q} u_{j}\right)-\right. \\
&\left.\left(u_{j}, \tilde{\tilde{\mathrm{L}}}_{Q} P_{0} \tilde{\tilde{\mathrm{L}}}_{Q} u_{j}\right) \frac{1}{(-i \nu)}\right] \\
&=\pi \frac{d}{d \mu}\left[\frac{1}{k} \sum_{j=1}^{k}\left(\tilde{\tilde{\mathrm{L}}}_{Q} u_{j}, P^{\prime}(\kappa-\delta, \mu) \tilde{\tilde{\mathrm{L}}}_{Q} u_{j}\right)\right]_{\mu=\kappa}
\end{aligned}
$$

and the theorem is proved.

From Theorem 5.3 we obtain

Theorem 5.4. Let $\kappa>\frac{1}{4}$ be an eigenvalue of $\Delta$ of multiplicity $k \geq 1$. Then

$$
\operatorname{Im} \hat{a}_{2}=\frac{1}{4 r} \frac{1}{k} \sum_{j=1}^{k} \sum_{l=1}^{m}\left|\left(\mathrm{~L}_{Q} u_{j}, \mathrm{E}_{l}\left(\frac{1}{2}+i r\right)\right)\right|^{2}
$$

where $\mathrm{E}_{l}\left(\frac{1}{2}+i r\right)$ is the Eisenstein series corresponding to the cusp $\mathrm{Z}_{l}$ at $\frac{1}{2}+i r$, and $\kappa=\frac{1}{4}+r^{2}$.

Proof. We prove (5.8) in 3 steps, first with $\mathrm{L}_{Q}$ replaced by $\tilde{\tilde{\mathrm{L}}}_{Q}$, then with $\mathrm{L}_{Q}$ replaced by $\tilde{\mathrm{L}}_{Q}$ and finally with $\mathrm{L}_{Q}$ itself.

1) By theorem 5.3 and (3.8)

$$
\operatorname{Im} \hat{a}_{2}=\frac{1}{2 r} \frac{1}{k} \sum_{j=1}^{k} \sum_{l=1}^{m}\left|\left(\tilde{\tilde{\mathrm{L}}}_{Q} u_{j}, \mathrm{E}_{l}\left(\frac{1}{2}+i r\right)\right)\right|^{2}
$$

2) Introducing (5.6) with $\lambda=1$ in (5.10), we obtain

$$
\begin{aligned}
\operatorname{Im} \hat{a}_{2}= & \frac{1}{2 r} \frac{1}{k} \sum_{j=1}^{k} \sum_{l=1}^{m} \mid\left(\tilde{\mathrm{L}}_{Q} u_{j}, \mathrm{E}_{l}\left(\frac{1}{2}+i r\right)\right)+ \\
& \frac{1}{2}\left(d^{\prime}(0) \Delta-\Delta d^{\prime}(0)\right) u_{j},\left.\mathrm{E}_{l}\left(\frac{1}{2}+i r\right)\right|^{2}
\end{aligned}
$$


Since $u_{j}$ is an eigenfunction and $\mathrm{E}_{l}\left(\frac{1}{2}+i r\right)$ a generalized eigenfunction of $\Delta$ with eigenvalue $\kappa=\frac{1}{4}+r^{2}$, we obtain, using that $d^{\prime}(0)$ has compact support,

$$
\begin{array}{r}
\left(\left(d^{\prime}(0) \Delta-\Delta d^{\prime}(0)\right) u_{j}, \mathrm{E}_{l}\left(\frac{1}{2}+i r\right)\right)= \\
\left(\Delta u_{j}, d^{\prime}(0) \mathrm{E}_{l}\left(\frac{1}{2}+i r\right)\right)-\left(d^{\prime}(0) u_{j}, \Delta \mathrm{E}_{l}\left(\frac{1}{2}+i r\right)\right)= \\
\kappa\left[\left(u_{j}, d^{\prime}(0) \mathrm{E}_{l}\left(\frac{1}{2}+i r\right)\right)-\left(d^{\prime}(0) u_{j}, \mathrm{E}_{l}\left(\frac{1}{2}+i r\right)\right)\right]=0
\end{array}
$$

Introducing (5.12) in (5.11), we get

$$
\operatorname{Im} \hat{a}_{2}=\frac{1}{2 r} \frac{1}{k} \sum_{j=1}^{k} \sum_{l=1}^{m}\left|\left(\tilde{\mathrm{L}}_{Q} u_{j}, \mathrm{E}_{l}\left(\frac{1}{2}+i r\right)\right)\right|^{2}
$$

3) Since $\sigma(\Delta(\varepsilon, \lambda))=\sigma\left(\tilde{\Delta}_{Q}(\varepsilon, \lambda)\right)$ for all $b$, the curves $\kappa(\varepsilon)$ and hence $\hat{a}_{2}$ are the same for $\Delta_{Q}(\varepsilon, \lambda)$ and $\tilde{\Delta}_{Q}(\varepsilon, \lambda)$ for all $b$. Thus $\operatorname{Im} \hat{a}_{2}$ as given by (5.13) is independent of $b$. In [P-S1] it is proved that

$$
\lim _{b \rightarrow \infty}\left|\left(\tilde{\mathrm{L}}_{Q} u_{j}, \mathrm{E}_{l}\left(\frac{1}{2}+i r\right)\right)\right|^{2}=\left|\left(\mathrm{L}_{Q} u_{j}, \mathrm{E}_{l}\left(\frac{1}{2}+i r\right)\right)\right|^{2}
$$

Since $\frac{1}{2 r} \frac{1}{k} \sum_{j=1}^{k} \sum_{l=1}^{m}\left|\left(\tilde{\mathrm{L}}_{Q} u_{j}, \mathrm{E}_{l}\left(\frac{1}{2}+i r\right)\right)\right|^{2}$ is constant equal to $\operatorname{Im} \hat{a}_{2}$, it follows that

$$
\operatorname{Im} \hat{a}_{2}=\frac{1}{2 r} \frac{1}{k} \sum_{j=1}^{k} \sum_{l=1}^{m}\left|\left(\mathrm{~L}_{Q} u_{j}, \mathrm{E}_{l}\left(\frac{1}{2}+i r\right)\right)\right|^{2}
$$

and the theorem is proved. 


\section{Appendix 1. The essential spectrum of $\Delta(\lambda)$.}

In this appendix we give the proof of 1 of Theorem 1.1. The self-adjoint operator

$$
A_{l}=-y^{2} \frac{d^{2}}{d y^{2}}+(2 \pi l)^{2} y^{2} \text { in } \mathrm{L}^{2}\left(a, \infty ; y^{-2} d y\right)
$$

defined by the boundary condition $f(a)=0$ is unitarily equivalent via the transformation $V$ defined by

$$
f(y) \rightarrow g(t)=(V f)(t)=f\left(e^{t}\right) e^{-t / 2}
$$

to the operator

$$
V A_{l} V^{-1}=-\frac{d}{d t^{2}}+(2 \pi l)^{2} e^{2 t} \text { in } \mathrm{L}^{2}(b, \infty: d t)
$$

with boundary condition $g(b)=0$, where $b=$ in $a$. This operator and hence $A_{l}$ has discrete spectrum.

It follows from (1.3)-(1.5), that the operators $\mathrm{L}^{j}(\lambda), j=1, \ldots, m$, in $\mathrm{L}^{2}\left(a_{j}, \infty ; y_{j}^{-2} d y_{j}\right)$ defined by the boundary conditions $f\left(a_{j}\right)=0$ have the essential spectrum $\frac{1}{4}+\lambda^{-2} \overline{\mathbb{R}^{+}}$. This can be seen as follows. A point $z$ is in $\sigma_{e}\left(\mathrm{~L}^{j}(\lambda)\right)$ if there exists a singular sequence $\left\{f_{n}\right\}$ for $\mathrm{L}^{j}(\lambda)$ at $z$, i.e. $\left\{f_{n}\right\} \subset \mathcal{D}\left(\mathrm{L}^{j}(\lambda)\right),\left\|f_{n}\right\|=1,\left\{f_{n}\right\}$ has no convergent subsequence and $\left\|\left(L^{j}(\lambda)-z\right) f_{n}\right\|_{n \rightarrow \infty} \rightarrow 0$. Let $\chi \in C^{\infty}\left(a_{j}, \infty\right)$ with $\chi\left(y_{j}\right)=0$ for $y_{j} \leq R_{j}, \chi\left(y_{j}\right)=1$ for $y_{j} \geq R+1$. Then $\chi\left(f_{n}\right)\left\|\chi f_{n}\right\|^{-1}$ is also a singular sequence for $\Delta^{j}(\lambda)$ at $z$ and also by (1.5) a singular sequence for the operator $L^{j}$ (with boundary condition $\left.y\left(a_{j}\right)=0\right)$ at $\left(z-\frac{1}{4}\right) \lambda^{2}+\frac{1}{4}$. Since $\sigma_{e}\left(\mathrm{~L}^{j}\right)=\left[\frac{1}{4}, \infty\right)$, it follows that $\sigma_{e}\left(\mathrm{~L}^{j}(\lambda)\right)=\frac{1}{4}+\lambda^{-2} \overline{\mathbb{R}^{+}}$.

From the above we prove that $\Delta(\lambda)$ has the essential spectrum $\sigma_{e}(\lambda)=$ $\frac{1}{4}+\lambda^{-2} \overline{\mathbb{R}^{+}}$The inclusion $\sigma_{e}(\lambda) \supseteq \sigma_{e}\left(\mathrm{~L}^{j}(\lambda)\right)$ is seen as follows. Let $\left\{f_{n}\right\}$ be a singular sequence for $\mathrm{L}^{j}(\lambda)$ at $\mu \in \sigma_{e}\left(\mathrm{~L}^{j}(\lambda)\right)$ and let $\chi$ be a function as defined above. Then $g_{n}=\left(\chi f_{n}\right)\left\|\chi f_{n}\right\|^{-1}$ is a singular sequence for $\mathrm{L}^{j}(\lambda)$ at $\mu$, which by extension to the rest of $M$, setting $g_{n}(z)=0$ for $z \notin\left\{z_{j} \in Z_{j} \mid\right.$ $\left.y_{j} \geq R\right\}$, becomes a singular sequence for $\Delta(\lambda)$ at $\mu$, hence $\mu \in \sigma_{e}(\Delta(\lambda))$.

Conversely, if $\mu \in \sigma_{e}(\Delta(\lambda))$ and $\left\{g_{n}\right\}$ is a singular sequence for $\Delta(\lambda)$ at $\mu$, let $\eta \in C^{\infty}(M)$ with $\eta(z)=0$ for $z \in M_{0}$ and $z \in Z_{j}$ with $y_{j} \leq R, \eta(z)=1$ for $y_{j} \geq R+1, j=1, \ldots, m$. Then $f_{n}=\left(\eta g_{n}\right)\|\eta g\|^{-1}$ is a singular sequence for $\Delta(\lambda)$ at $\mu$, and it follows that at least in one cusp $Z_{j},\left\{f_{n}\right\}$ is a singular sequence for $\Delta_{j}(\lambda)$. Since $A_{j}^{l}$ has discrete spectrum for $l \in \mathbb{Z} /\{0\}$, we must have $\sigma_{e}\left(\Delta_{j}(\lambda)\right)=\sigma_{e}\left(\mathrm{~L}^{j}(\lambda)\right)$, so $\mu \in \sigma_{e}\left(\mathrm{~L}^{j}(\lambda)\right)$. 


\section{Appendix 2. A dense set of analytic vectors.}

Theorem A2. Let $M$ be an admissible manifold $M=M_{0} \cup Z_{1} \cup \cdots \cup Z_{m}$ with $m$ cusps, and let $\left\{U_{\lambda}\right\}_{\lambda \in(1-\alpha, \infty)}$ be the family of power transformations defined in section 1 . There exists a dense set $\mathcal{F}$ of vectors $f$ on $\mathrm{L}^{2}(M)$, such that the functions $f(\lambda)=U_{\lambda} f$ have analytic extensions to

$$
\left.G_{\alpha}^{\prime}=\left\{\lambda \in G_{\alpha} \mid \operatorname{Re} \lambda>0\right\}=\{\lambda \in \mathbb{C} \mid \operatorname{Re} \lambda>0\} \backslash(0,1-\alpha]\right\}
$$

and for each $\lambda \in G_{\alpha}^{\prime}$ the set $\{f(\lambda) \mid f \in \mathcal{F}\}$ is dense in $\mathrm{L}^{2}(M)$.

Proof. To simplify notation, consider the case of a manifold $M=M_{0} \cup Z$ with one cusp. Let $f \in \mathrm{L}^{2}(M)$ and let $\eta \in C^{\infty}\left(\mathbb{R}^{+}\right)$be a function satisfying

$$
0 \leq \eta(y) \leq 1, \eta^{\prime}(y) \geq 0, \eta(y)=\left\{\begin{array}{l}
1 \text { for } K \leq y<\infty \\
0 \text { for } 0<y \leq K-1
\end{array}\right.
$$

assuming $K \geq a+1$. Let

$$
\tilde{\eta}(z)=\left\{\begin{array}{l}
\eta(y) \text { for } z=x+i y \in Z \\
0 \text { for } z \in M_{0}
\end{array}\right.
$$

Let $f_{1}=f(1-\tilde{\eta}), f_{2}=f \tilde{\eta}$. Consider $\mathcal{P}$ of all polynomials $P$ in $y^{-1}$ of the form

$$
P_{n}\left(y^{-1}\right)=\sum_{k=0}^{n} a_{k} y^{-k}, \quad n=0,1,2, \ldots
$$

For $y \geq K-1$, write

$$
f(x, y)=a_{0}(y)+\sum_{n}^{\prime} a_{n}(y) e^{2 \pi i n x}
$$

$\mathcal{P}$ is dense in $\mathrm{L}^{2}\left(K-1, \infty: y^{-2} d y\right)$; a function $g \in \mathrm{L}^{2}\left(K-1, \infty ; y^{-2} d y\right)$ can be approximated by a function $h \in C_{c}(K-1, \infty) ; h\left(y^{-1}\right) \in C_{c}\left(0,(K-1)^{-1}\right)$ can be approximated uniformly by a polynomial $P_{n}(y)$ and hence $h$ by $P_{n}\left(y^{-1}\right)$ uniformly and therefore in $\mathrm{L}^{2}\left(K-1, \infty ; y^{-2} d y\right)$, so $g$ is approximated in $\mathrm{L}^{2}\left(K-1, \infty ; y^{-2} d y\right)$, by $P_{n}\left(y^{-1}\right) \in \mathcal{P}$.

Let $p_{\delta} \in \mathcal{P}$ be such that

$$
\left\|a_{0}-p_{\delta}\right\|_{\mathrm{L}_{\left(K-1, \infty ; y^{2} d y\right)}^{2}}<\delta
$$


Then

$$
\left\|a_{0} \eta-p_{\delta} \eta\right\|_{\mathrm{L}_{\left(K-1, \infty ; y^{-2} d y\right)}^{2}}<\delta
$$

hence

$$
\left\|f_{2}-\left(p_{\delta} \eta+\sum_{n}^{\prime} a_{n}(y) \eta(y) e^{2 \pi i n x}\right)\right\|_{L^{2}\left(K-1, \infty ; y^{-2} d y\right)}=
$$

so

$$
\begin{aligned}
&\left\|f-\left[f_{1}+\tilde{p}_{\delta} \tilde{\eta}+\sum_{n}^{\prime} a_{n}(y) \tilde{\eta}(y) e^{2 \pi i n x}\right]\right\|_{L^{2}(M)}= \\
&\left\|a_{0} \eta-p_{\delta} \eta\right\|_{\left.L_{(K-1, \infty ; y}^{2}-2 d y\right)}<\delta
\end{aligned}
$$

where $\tilde{p}_{\delta}$ is $p_{\delta}$ extended by 0 to $M$. Thus the set of functions

$$
\mathcal{F}=\left\{f_{p_{\delta}}=f_{1}+\tilde{p}_{\delta} \tilde{\eta}+\sum_{n}^{\prime} a_{n}(y) \tilde{\eta}(y) e^{2 \pi i n x} \mid f \in \mathrm{L}^{2}(M)\right\}
$$

is dense in $\mathrm{L}^{2}(M)$.

We shall prove that every $f_{p_{\delta}} \in \mathcal{F}$ is analytic with respect to $U_{\lambda}$ in $G_{\alpha}^{\prime}$.

For $y \leq K,\left(U_{\lambda} f\right)(x, y)=f(x, y)$. For $y \geq K, f_{1}(x, y)=0$, so $U_{\lambda} f_{1}=f_{1}$. By definition

$$
U_{\lambda}\left(\sum_{n}^{\prime} a_{n}(y) \eta(y) e^{2 \pi i n x}\right)=\sum_{n}^{\prime} a_{n}(y) \eta(y) e^{2 \pi i n x}
$$

Moreover, $\left.U_{\lambda} \tilde{p}_{\delta} \tilde{\eta}\right)=\left(\widetilde{U_{\lambda}^{0}\left(p_{\delta} \eta\right.}\right)$, where

$$
\left(U_{\lambda}^{0}\left(p_{\delta} \eta\right)\right)(y)=\left(\psi_{\lambda}^{\prime}(y)\right)^{\frac{1}{2}} y^{-(\lambda-1) \varphi(y)} p_{\delta}\left(y^{1+(\lambda-1) \varphi(y)}\right) \eta\left(y^{1+(\lambda-1) \varphi(y)}\right)
$$

We show that

$$
\eta\left(y^{1+(\lambda-1) \varphi(y)}\right)=\eta(y) \text { for } 1-\alpha<\lambda<\infty
$$

For $y \geq K, y^{1+(\lambda-1) \varphi(y)} \geq K$, since $\psi(y)=y^{1+(\lambda-1) \varphi(y)}$ is increasing $\left(\psi^{\prime}(y)>0\right.$ ) and $\psi(K)=K$. Since $\eta(y)=1$ for $y \geq K$, we 
have $\eta\left(y^{1+(\lambda-1) \varphi(y)}\right)=1=\eta(y)$. For $y<K, y^{1+(\lambda-1) \varphi(y)}=y$ so $\eta\left(y^{1+(\lambda-1) \varphi(y)}\right)=\eta(y)$. Putting this together, we get (1). Moreover

$$
p_{\delta}\left(y^{1+(\lambda-1) \varphi(y)}\right)=\sum_{k=0}^{n} a_{k} y^{-k(1+(\lambda-1) \varphi(y))}
$$

For $\lambda \in G_{\alpha}^{\prime}, p_{\delta}\left(y^{1+(\lambda-1) \varphi(y)}\right)$ is bounded for $0<\delta \leq y<\infty$ and analytic for $y>0$. Finally

$$
\left(\psi_{\lambda}^{\prime}(y)\right)^{\frac{1}{2}} y^{-(\lambda-1) \varphi(y)}=y^{-(\lambda-1) \varphi(y)}\left(1+(\lambda-1)\left(\varphi(y)+\varphi^{\prime}(y) y \log y\right)\right)
$$

is analytic for $\lambda \in G_{\alpha}^{\prime}$ with values in $\mathrm{L}^{2}\left(\delta, \infty ; y^{-2} d y\right)$.

It follows that $U_{\lambda}^{0}\left(p_{\delta} \eta\right)=\left(\psi_{\lambda}^{\prime}(y)\right)^{\frac{1}{2}} y^{-(\lambda-1) \varphi(y)} p_{\delta}\left(y^{1+(\lambda-1) \varphi(y)}\right) \eta(y)$ is analytic in $G_{\alpha}^{\prime}$ with values in $\mathrm{L}^{2}\left(\delta, \infty ; y^{-2} d y\right)$ and hence

$$
U_{\lambda} f_{p_{\delta}}=f_{1}+\left(\widetilde{U_{\lambda}^{0}\left(p_{\delta} \eta\right)}\right)+\sum_{n}^{\prime} a_{n}(y) \eta(y) e^{2 \pi i n x}
$$

is analytic in $G_{\alpha}^{\prime}$ with values $\mathrm{L}^{2}(M)$.

The density of $\left\{U_{\lambda} f_{p_{\delta}}\right\}$ in $\mathrm{L}^{2}(M)$ for each $\lambda \in G_{\alpha}^{\prime}$ is established by a proof similar to that given above for $\lambda=1$.

For a manifold $M=M_{0} \cup Z_{1} \cup \cdots \cup Z_{m}$ with $m$ cusps, the proof is similar. We approximate in each cusp the 0 -th Fourier component by a polynomial in $y_{j}^{-1}$, and a function $f$ in $\mathrm{L}^{2}(M)$ is approximated by a function $\tilde{f}$ equal to $f$ on $M_{0}$ and in the proximate ends of the cusps and with the 0 -the Fourier component equal to suitable polynomial in $y_{j}^{-1}$ in the distant end of each $\operatorname{cusp} Z_{j}$. 


\section{Appendix 3. The resolvent kernel.}

We consider the integral $I\left(\mu_{0}+i \varepsilon\right)$ given by $(3.4), \varepsilon>0$.

Fix $\delta$ with $\varepsilon<\delta<\mu_{0}-\frac{1}{4}$, let $C_{\delta}$ be the semicircle in the upper half-plane with center $\mu_{0}$ and radius $\delta$, oriented counter-clockwise, and let $\Gamma_{\delta}$ be the closed curve composed of the interval $\left[\mu_{0}-\delta, \mu_{0}+\delta\right]$ and $C_{\delta}$.

We write the integral as

$$
I\left(\mu_{0}+i \varepsilon\right)=\int_{\left|\mu-\mu_{0}\right| \geq \delta}+\int_{\Gamma_{\delta}}-\int_{C_{\delta}}=I_{1}(\varepsilon)+I_{2}(\varepsilon)+I_{3}(\varepsilon)
$$

We calculate the limits of $I_{1}(\varepsilon), I_{2}(\varepsilon)$ and $I_{3}(\varepsilon)$ as $\varepsilon \downarrow 0$, where $f$ and $g$ are fixed in $\mathrm{L}_{\beta}^{2}(M)$ for some $\beta>0$. Let

$$
\begin{aligned}
F_{f, g}(\mu)=\left(f, E_{j}\left(\bullet, \frac{1}{2}+\right.\right. & \left.\left.i\left(\mu-\frac{1}{4}\right)^{\frac{1}{2}}\right)\right) \\
& \left(E_{j}\left(\bullet, \frac{1}{2}+i\left(\mu-\frac{1}{4}\right)^{\frac{1}{2}}\right), g\right) \frac{1}{2}\left(\mu-\frac{1}{4}\right)^{-\frac{1}{2}}
\end{aligned}
$$

Then

$$
\begin{gathered}
\lim _{\varepsilon \downarrow 0} I_{1}(\varepsilon)=\int_{\left|\mu-\mu_{0}\right| \geq \delta}\left(\mu-\mu_{0}\right)^{-1} F_{f, g}(\mu) d \mu \\
I_{2}(\varepsilon)=\int_{\Gamma_{\delta}}\left(\mu-\mu_{0}-i \varepsilon\right)^{-1} F_{f, g}(\mu) d \mu=2 \pi i F_{f, g}\left(\mu_{0}+i \varepsilon\right) \\
\lim _{\varepsilon \downarrow 0} I_{2}(\varepsilon)=2 \pi i F_{f, g}\left(\mu_{0}\right)
\end{gathered}
$$

(3) $\lim _{\varepsilon \downarrow 0} I_{3}(\varepsilon)=-\int_{C_{\delta}}\left(\mu-\mu_{0}\right)^{-1}\left(F_{f, g}(\mu)-\right.$

$$
\left.-F_{f, g}\left(\mu_{0}\right)\right) d \mu-F_{f, g}\left(\mu_{0}\right) \int_{C_{\delta}}\left(\mu-\mu_{0}\right)^{-1} d \mu=I_{4}+I_{5}
$$


Since $F_{f, g}(\mu)$ is real analytic in $\mu$ near $\mu_{0}$, we have for $\varepsilon<\delta<\mu_{0}-\frac{1}{4}$ the estimate

$$
\left|I_{4}(\varepsilon)\right| \leq K \delta
$$

while

$$
I_{5}=-\pi i F_{f, g}\left(\mu_{0}\right)
$$

Letting $\delta \downarrow 0$, we obtain

$$
\lim _{\varepsilon \downarrow 0} I\left(\mu_{0}+i \varepsilon\right)=v \cdot p \cdot \int_{\frac{1}{4}}^{\infty}\left(\mu-\mu_{0}\right)^{-1} F_{f, g}(\mu) d \mu+\pi i F_{f, g}\left(\mu_{0}\right)
$$

Similarly, $\lim _{\varepsilon \downarrow 0} I\left(\mu_{0}-i \varepsilon\right)$ is given by (6) with $\pi i$ replaced by $-\pi i$.

From (3.3) and (6) we obtain for $f, g \in \mathrm{L}_{\beta}^{2}(M), f \in \mathcal{H}_{c}(\Delta)$

$$
\begin{aligned}
& \left(R\left(\mu_{0} \pm i 0\right) f, g\right):=\lim _{\varepsilon \downarrow 0}\left(R\left(\mu_{0} \pm i \varepsilon\right) f, g\right) \\
= & \frac{1}{2 \pi} \sum_{j=1}^{m}\left\{v \cdot p \cdot \int_{\frac{1}{4}}^{\infty}\left(\mu-\mu_{0}\right)^{-1} F_{f, g}(\mu) d \mu \pm \pi i F_{f, g}\left(\mu_{0}\right)\right\}
\end{aligned}
$$

Theorem A3. Let $\beta>0$ and $\kappa>0$ be given, and let $\tilde{R}\left(\frac{1}{2}+i t\right)$ be defined as an operator in $\mathcal{B}\left(\mathrm{L}_{\beta}^{2}(M), \mathrm{L}_{-\beta}^{2}(M)\right)$ by

$$
\tilde{R}\left(\frac{1}{2}+i t\right)= \begin{cases}R\left(\frac{1}{2}+i t\right) & \operatorname{Im} t<0 \\ R\left(\frac{1}{4}+t^{2} \pm i 0\right) & \text { for } t \in \mathbb{R}^{\mp}\end{cases}
$$

Then $\left\|\tilde{R}\left(\frac{1}{2}-i t\right)\right\|<C$ on $\{t \mid \operatorname{Im} t<0\},|t| \geq \kappa$.

Proof. Let $0<\delta<\frac{1}{2}\left(\frac{1}{4}+\kappa^{2}\right)$ be fixed. We use expressions (1)-(3) to estimate the different terms of $\left(\tilde{R}\left(\frac{1}{2}+i t\right) f, g\right)$ for $f, g \in \mathrm{L}_{\beta}^{2}(M)$ and $t \in[\kappa, \infty]$, setting $\mu_{0}=\frac{1}{4}+t^{2}$.

We obtain from

$$
I_{1}(0)=\lim _{\varepsilon \downarrow 0} I_{1}(\varepsilon)=\int_{\left|\mu-\mu_{0}\right| \geq \delta}\left(\mu-\mu_{0}\right)^{-1} F_{f, g}(\mu) d \mu
$$




$$
\begin{aligned}
&\left|I_{1}(0)\right| \leq \delta^{-1} \frac{1}{2}\left(\mu_{0}-\delta\right)^{-\frac{1}{2}}\left(\int_{0}^{\infty}\left|\left(f, E_{j}\left(\bullet, \frac{1}{2}+i t\right)\right)\right|^{2} d t\right)^{\frac{1}{2}} \\
&\left(\int_{0}^{\infty}\left|\left(E_{j}\left(\bullet, \frac{1}{2}+i t\right), g\right)\right|^{2} d t\right)^{\frac{1}{2}} \\
& \leq \delta^{-1} \frac{1}{2}\left(\mu_{0}-\delta\right)^{-\frac{1}{2}}\|f\|_{L^{2}(M) \cdot} \cdot\|g\|_{L^{2}(M)} \leq C \cdot\|f\|_{L_{\beta}^{2}(M)} \cdot\|g\|_{L_{\beta}^{2}(M)} \\
&\left|I_{2}(0)\right| \leq C \cdot\|f\|_{L_{\beta}^{2}(M)} \cdot\|g\|_{L_{\beta}^{2}(M)}\left\|E_{j}\left(\bullet, \frac{1}{2}+i\left(\mu_{0}-\frac{1}{4}\right)^{\frac{1}{2}}\right)\right\|_{L_{-\beta}^{2}(M)}^{2} \\
&\left|I_{3}(0)\right| \leq \delta^{-1} \cdot\|f\|_{L_{\beta}^{2}(M)} \cdot\|g\|_{L_{\beta}^{2}(M)} \cdot \max _{\mu \in C_{\delta}}\left\|E_{j}\left(\bullet, \frac{1}{2}+i\left(\mu-\frac{1}{4}\right)^{\frac{1}{2}}\right)\right\|_{L_{-\beta}^{2}}^{2}
\end{aligned}
$$

and the theorem follows.

\section{References.}

[B-C] Balslev, E. and Combes, J. M.: Spectral properties of many body Schrödinger operators with dilation-analytic interactions, Comm. Math Phys. Vol. 22, 1971, 280-294.

[CdV] de Verdiere, Y. Colin: Pseudo-Laplacians II, Ann. Inst. Fourier 33, 2, $1983,87-113$

[F] Faddeev, L. D.: Expansion in eigenfunctions of the Laplace operator on the fundamental domain of a discrete group on the Lobacevskii plane, AMS Trans. Trudy, 1967, 357-386.

[Hu] Hunziker, W.: Distortion analycity and molecular resonance curves, Ann. Inst. H Poincaré, Vol. 48, 4, 1986, 339-358.

[K] Kato, T.: Perturbation Theory for Linear Operators, Springer Verlag, 1976.

[L-P] Lax, P. and Phillips, R.: Scattering Theory for automorphic functions, Annals of Math. Studies, Princeton University Press, 1976.

[M] Müller, W.: Spectral geometry and scattering theory for certain complete surfaces of finite volume, Invent. Math. 109, 1992, 265-305.

[Pe1] Petridis, Y.: Spectral data for finite volume hyperbolic surfaces at the bottom of the continuous spectrum, J. of Functional Analysis, 124, 1994, 1, 61-94. 
[Pe2] Petridis, Y.: On the singular set, the resolvent and Fermi's Golden Rule for finite volume hyperbolic surfaces, Manuscript Math, 82, 1994, $3-4,331-347$.

[P-S1] Phillips, R. and Sarnak, P.: On cusp forms for cofinite subgroups of PSL $(2, \mathbb{R})$, Invent. Math. 80, 1985, 339-364.

[P-S2] Phillips, R. and Sarnak, P.: The Weyl Theorem and the deformation of Discrete groups, Comm. Pure and Appl. Math., Vol. 38, 1985, 853-866.

[P-S3] Phillips, R. and Sarnak, P.: Perturbation Theory for the Laplacian on automorphic functions, Journal of the AMS, Vol. 5, No. 1 1992, 1-32.

[Se] Selberg, A.: Harmonic Analysis, in Collected Papers, Vol. I, 626-674, Springer Verlag, 1989.

[Si] Simon, B.: Resonances in $N$-body Quantum systems and the Foundations of time dependent perturbation theory, Annals of Math., Vol. 97, $1973,247-274$.

Acknowledgement. I would like to thank R. Phillips and P. Sarnak for helpful conversations and $\mathrm{M}$. Zworski for suggesting the idea of transforming only in the 0-the Fourier mode.

RECeived SEPTEMBer 9, 1994.

\author{
AARHUS UNIVERSITÄT \\ INSTITUT FOR MATEMATISKE FAG \\ Ny MUNKEGADE \\ 8000 Aarhus C, DeNMARK \\ BALSLEV@MI.AAU.DK
}

